\title{
THE ENGINEER GRAPPLES WITH NONLINEAR PROBLEMS ${ }^{1}$
}

\author{
THEODORE VON KÁRMÁN
}

I do not believe that one could connect justly the name of Gibbs with practical applications of applied mathematics, for his main interest was certainly centered on basic conceptions of mathematical physics. Nevertheless, for example, his beautiful work on graphical methods in thermodynamics is a brilliant example of the presentation of theoretical relations in a form which appeals to the engineer.

This lecture is intended as an effort to improve the convergence between the viewpoints of mathematics and engineering. Thus, I feel it is not inappropriate to dedicate it to the memory of Josiah Willard Gibbs.

Engineering mathematics is generally considered as a collection of mathematical methods adapted for the solution of relatively simple problems. These problems of ten might require lengthy numerical calculations or graphical constructions, but supposedly can be worked out without the use of advanced methods of mathematical analysis. This description was perhaps correct some decades ago; today a large group of scientific workers is engaged in applying various methods of classical and modern analysis to problems in electrical, civil, mechanical, aeronautical and also chemical engineering. It is not possible to give an exhaustive list of all types of problems which require the applications of advanced analytical methods. In the following table merely some of the most important engineering problems and the mathematical concepts and methods involved in their treatment are indicated:

Associated TOPICS OF ENGINEERING AND MATHEMATICS

\begin{tabular}{c|c}
\hline \hline Mathematical topics & Engineering problems \\
\cline { 2 - 2 } $\begin{array}{l}\text { Vector algebra, systems of linear } \\
\text { equations. Tensors and matrices. Alge- } \\
\text { braic equations. Ordinary differential } \\
\text { equations with given initial conditions. } \\
\text { Elementary operational calculus. }\end{array}$ & $\begin{array}{c}\text { Engine dynamics, vibration of sys- } \\
\text { tems with a finite number of degrees of } \\
\text { freedom. Rotating electric machinery. }\end{array}$ \\
\hline $\begin{array}{l}\text { Ordinary differential equations and } \\
\text { their boundary problems. Eigenvalues } \\
\text { and eigenfunctions. Expansion in or- }\end{array}$ & $\begin{array}{c}\text { Equilibrium, buckling and harmonic } \\
\text { vibrations of beams. Critical frequencies } \\
\text { and speeds. One-dimensional problems }\end{array}$
\end{tabular}

${ }^{1}$ The fifteenth Josiah Willard Gibbs Lecture, delivered at Columbus, Ohio, December 27, 1939, under the auspices of the American Mathematical Society with the cooperation of the American Association for the Advancement of Science. 


\begin{tabular}{l|c}
\hline \hline \multicolumn{1}{c|}{ Mathematical topics } & \multicolumn{1}{c}{ Engineering problems } \\
\hline $\begin{array}{l}\text { thogonal functions. Calculus of varia- } \\
\text { tions of simple integrals. }\end{array}$ & $\begin{array}{l}\text { of stationary flow of matter, ele tricity } \\
\text { and heat. }\end{array}$ \\
\hline $\begin{array}{c}\text { Functions of complex variables. Con- } \\
\text { formal transformation. Logarithmic po- } \\
\text { tential. }\end{array}$ & $\begin{array}{c}\text { Two-dimensional velocity, electro- } \\
\text { magnetic and temperature fields. }\end{array}$ \\
$\begin{array}{c}\text { Vector and tensor analysis. Partial } \\
\text { differential equations of the elliptic type } \\
\text { and their boundary problems. Expansion } \\
\text { in orthogonal functions. Integral equa- } \\
\text { tions. Calculus of variations of multiple } \\
\text { integrals. }\end{array}$ & $\begin{array}{l}\text { Stress distribution in elastic bodies; } \\
\text { equilibrium, buckling and harmonic vi- } \\
\text { bration of plates and shells. Three-di- } \\
\text { mensional flow and field problems. }\end{array}$ \\
$\begin{array}{c}\text { Partial differential equations of the } \\
\text { parabolic and hyperbolic type. Advanced } \\
\text { operational calculus. }\end{array}$ & $\begin{array}{l}\text { Transient phenomena of heat con- } \\
\text { duction and wave propagation (variable } \\
\text { flow in channels, water hammer in pipes, } \\
\text { surges in cables, waves in solids and } \\
\text { fluids, electromagnetic waves). }\end{array}$ \\
\hline
\end{tabular}

Though most of the various branches of mathematical analysis appear in this table, it does not follow that the engineer has to master, for example, the complete theory of linear differential and integral equations or the calculus of variations. $\mathrm{He}$ is mostly interested in methods of obtaining approximate solutions. However, it is interesting to note that the methods which are perhaps the most powerful tools of the mathematician for proofs of existence of solutions have been proved to be the most helpful in obtaining numerical approximations, for example, step-by-step integration, iteration, successive approximation, direct methods of finding maxima and minima. The iteration method is known to most engineers as the Stodola or Vianello method. The direct methods of the calculus of variations are known as the energy method, Rayleigh-Ritz method, Galerkin method, and so on.

The problems listed in the above table provide a happy hunting ground to the mathematician who is interested in applying his science for practical purposes. We know that not all mathematicians have this interest; a great British mathematician is credited with the remark that his field - the theory of numbers - is the only field of mathematics that, fortunately, has not been prostituted by being useful to some practical end. Others believe that the engineering applications of mathematics do not require ingenuity in the mathemati- 
cal sense; however, there are many engineering problems that are far from being an easy mark for the hunter. Most of the problems compiled above are readily accessible to the methods of mathematical analysis because they are linear problems. In many cases the engineer can linearize his problem by means of simplifying assumptions and a mathematical text will easily supply him with all the help he needs. However, if the engineer has a real nonlinear problem, that is, one which loses its sense by linearization, very of ten he has to grapple with it by himself.

In this paper the attempt is made to give a review of nonlinear problems which are of practical interest and for whose solution methods have been worked out or at least suggested by mathematicians, physicists or engineers. The review is not exhaustive, but it was attempted to include examples of the most typical problems. The question of existence and uniqueness of solutions is only superficially touched; emphasis is laid on methods for obtaining approximate solutions. Many physicists and engineers have the feeling that so-called mathematical proofs of existence and uniqueness of solutions very of ten merely verify facts which are more or less evident from physical considerations. Unfortunately, in most nonlinear problems physical reasoning is not sufficient or not fully convincing, so that in these cases the questions of existence and uniqueness represent a real challenge to the mathematician. Certain investigations of $\mathrm{H}$. Poincaré and $\mathrm{T}$. Levi-Civita can be mentioned as examples in which mathematical reasoning has virtually extended the range of physical vision. The modern methods of function spaces are likely to make essential contributions to the problem of existence and uniqueness of solutions of nonlinear equations and their boundary problems. Promising steps in this direction have already been made. However, the writer is afraid that this aspect of the subject is beyond his own competency and the scope of this paper.

1. Nonlinear oscillations. General remarks. The theory of mechanical oscillations commonly used in engineering practice is based on the assumption that the restoring forces are proportional to the deflections and the damping forces to the velocities, that is, to the first derivatives of the deflections with respect to time. If we assume constant masses, the equations of motion of an oscillating system are linear, containing as coefficients constant inertia, damping and spring factors. We obtain analogous equations for the oscillations of an electric circuit by assuming constant impedances, that is, by assuming that the network contains only constant reactances, resistances and 
capacities. The oscillations governed by linear equations may be called "linear oscillations."

Let us consider the oscillations of a single mass $m$ assuming that the deflection $x$ produces a restoring force $-k x$ and the damping force is equal to $-\beta \dot{x}$, where $\dot{x}=d x / d t=v$ is the velocity of the mass. Then the equation of motion for the free oscillations of this mass is

$$
m \ddot{x}+k x+\beta \dot{x}=0 .
$$

Introducing the natural frequency $\omega$ of the undamped oscillations, that is, the frequency for the case $\beta=0$, we have

$$
\ddot{x}+\omega^{2} x+\frac{\beta}{m} \dot{x}=0 .
$$

We will generalize equation (1.2) in the form

$$
\ddot{x}+\omega^{2} x=f(x, \dot{x}) .
$$

This general form of the equation of free oscillation includes the cases of nonlinear damping and nonlinear restoring force. Often the deviation from linearity causes only a small deviation of the linear oscillation without changing the general character of the motion. For example, the simple harmonic oscillation, which we would obtain in the linear case, will be accompanied by harmonics of small amplitude if the deviation from linearity is taken into account. However, in some cases the whole character of the oscillatory motion changes. Slight negative damping in the range of small deflections may cause "self-excited periodic oscillations," whose period is quite different from the period of the undamped harmonic oscillation. Nonlinearity of the spring factor may fundamentally change the resonance phenomena, that is, the response of the system to external periodic forces. In the case of a pure linear system the response to a periodic force consists of a forced oscillation whose period is equal to that of the force and of free oscillations, whose period is the natural period of the system. If the two frequencies coincide, resonance occurs. One can easily visualize that in a nonlinear case the periodic force will excite also higher harmonics, that is, oscillations whose frequencies are multiples of its own frequency. It is more difficult to see offhand that in such a system an external force can also excite oscillations whose frequency is a fraction of its own frequency. This phenomenon is known as "subharmonic resonance." The mathematical analysis of the phenomena of self-excited oscillations and subharmonic resonance makes use of certain methods developed by $\mathrm{H}$. Poincaré in connection 
with his astronomical investigations and shall be discussed briefly in the next two sections.

2. Self-excited nonlinear oscillations (relaxation oscillations). Let us assume that the function $f(x, \dot{x})$ in equation (1.3) has the form $f(x, \dot{x})=\mu \phi(x) \dot{x}$, where $\mu$ is a positive constant parameter. It is seen that in this case the damping is proportional to the velocity $v=\dot{x}$, but the magnitude of the damping factor is in general a function of the deflection. The equation (1.3) in this case takes the form

$$
\ddot{x}+\omega^{2} x=\mu \phi(x) \dot{x} .
$$

Equation (2.1) can be reduced to a differential equation of the first order by considering $\dot{x}=v$ as function of $x$. We obtain

$$
\frac{d v}{d x}=-\omega^{2} \frac{x}{v}+\mu \phi(x) .
$$

This equation can be solved by the method of isoclines. Consider first the case $\mu=0$. The integral curves of (2.2) are in this case concentric ellipses, given by

$$
v^{2}+\omega^{2} x^{2}=\text { const. }
$$

One of these ellipses is shown in Fig. 1a. Since $d t=d x / v$, we obtain the $x, v$ values corresponding to the actual motion by proceeding clockwise along the integral curves. The corresponding motion is a harmonic oscillation with constant amplitude.

Let us now assume that $\phi(x)=1$, that is, the damping factor is constant. It is known that the resulting motion is a harmonic oscillation with decreasing amplitude if $\mu<0$ and increasing amplitude if $\mu>0$. We obtain logarithmic spirals, which in the stable case $(\mu<0)$ converge toward the origin (Fig. 1b) and in the unstable case $(\mu>0)$ diverge from the origin (Fig. 1c).

We shall now assume that $\phi(x)$ is variable and investigate the existence of periodic solutions. In the $x, v$ plane a periodic solution appears as a closed curve. Let us multiply both sides of equation (2.2) by $v d x$ and integrate along the closed curve. Then we see that $\mu \mathscr{\Phi} \phi(x) v d x=\mu \Phi \phi(x) v^{2} d t=0$. Hence $\phi(x)$ must change its sign during the motion. In other words, to obtain a periodic motion it is a necessary condition that the damping factor change its sign as function of the deflection.

Van der Pol studied in detail an interesting special case assuming that $\phi(x)=1-x^{2}$. It is seen that for $x<1$ we have negative damping 
which becomes positive when $x>1$. Fig. 2 and Fig. 3 show integral curves in the $x, v$ plane for $\mu=0.1$ and $\mu=1.0$ respectively. The in-

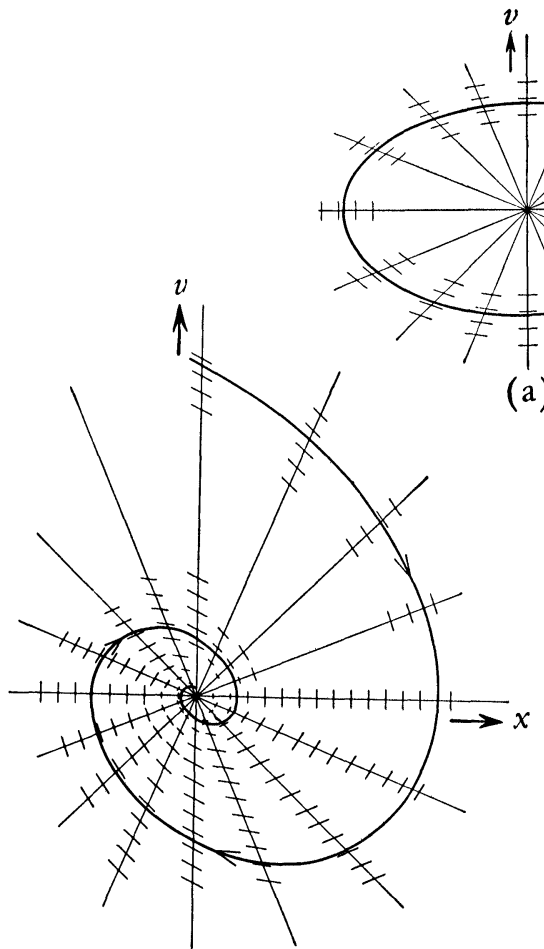

(b)

(a)
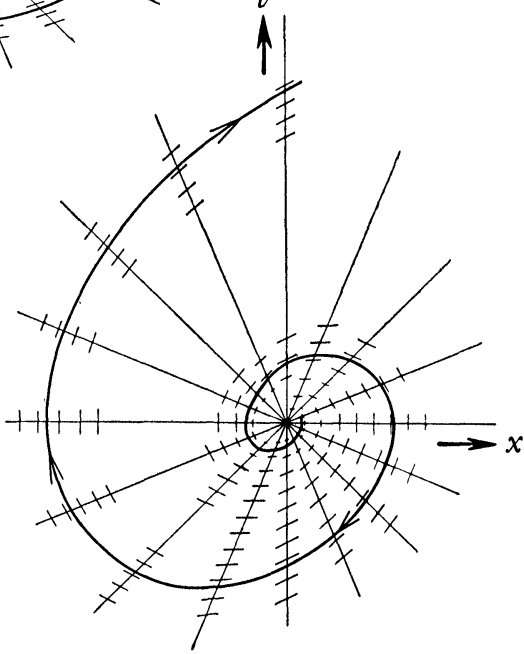

(c)

Fig. 1a, b, c

tegral curves near the origin have the shape of logarithmic spirals, but instead of diverging to infinity they converge to a closed curve. The integral curves originating at infinity converge to the same closed curve, which represents a periodic motion with constant amplitude. For small values of $\mu$, for example, $\mu=0.1$, the closed curve is only slightly different from an ellipse, but its shape varies quite radically with increasing $\mu$. The corresponding motions for $\mu=0.1,1.0$ and 10 are shown in Fig. 4 where the deflection is plotted as function of time. It is seen that for $\mu=0.1$ the motion is very nearly harmonic, whereas for $\mu=10$ the oscillation is made up of sudden transitions between deflections of opposite sign. The period of the harmonic motion is de- 
termined by the spring factor and the mass. The period of the motion corresponding to $\mu=10$ is much larger and depends both on $\omega$ and $\mu$.

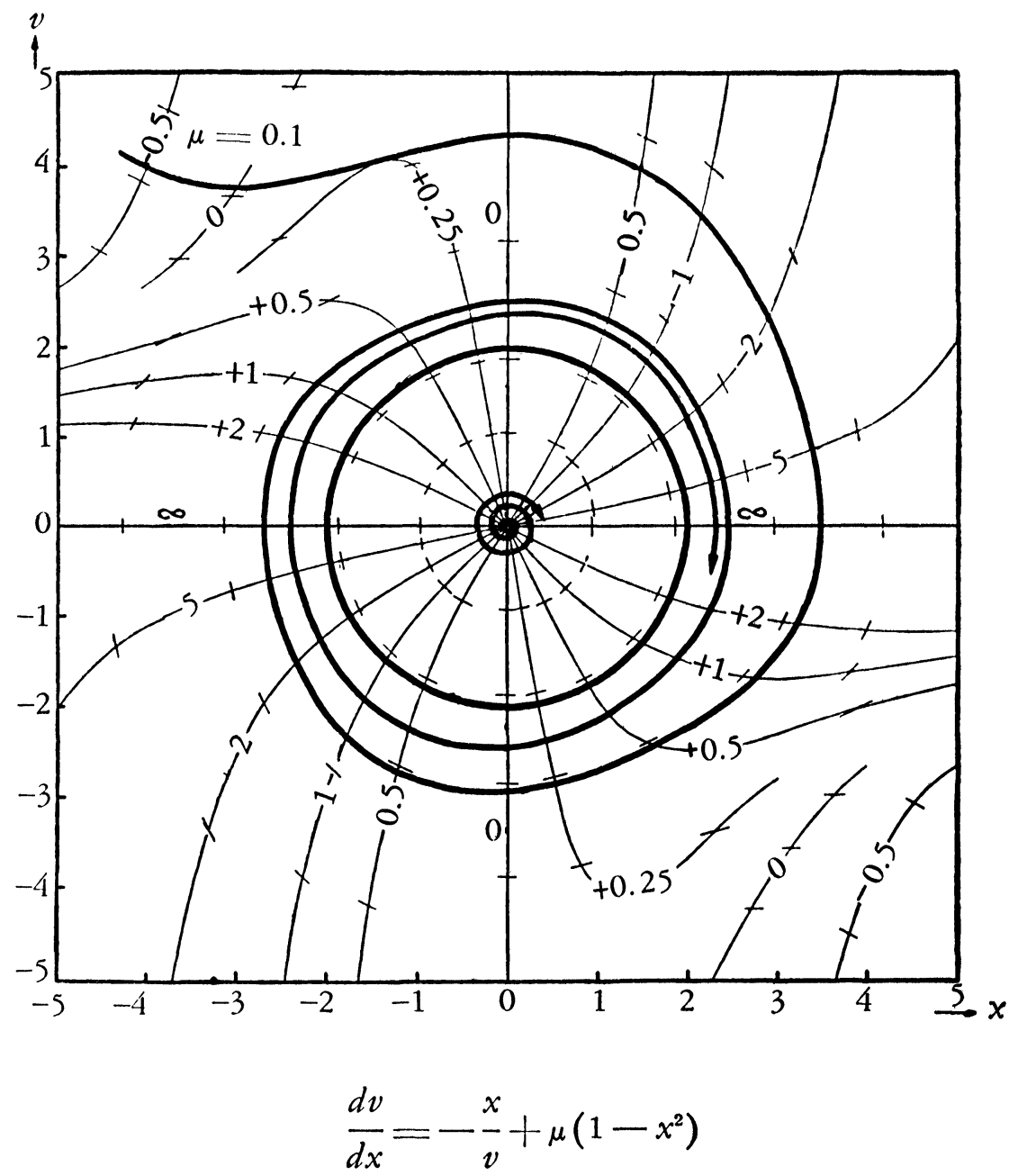

FIG. 2

From B. Van der Pol, Philosophical Magazine, vol. 2 (192ú)

For this reason Van der Pol calls this type of motion a relaxation oscillation. The term self-excited oscillation is also often used. The physically interesting point is that we obtain a periodically undamped oscillation in a damped mechanical or electrical system because the 
damping coefficient is variable and for small deflections has a slight negative value.

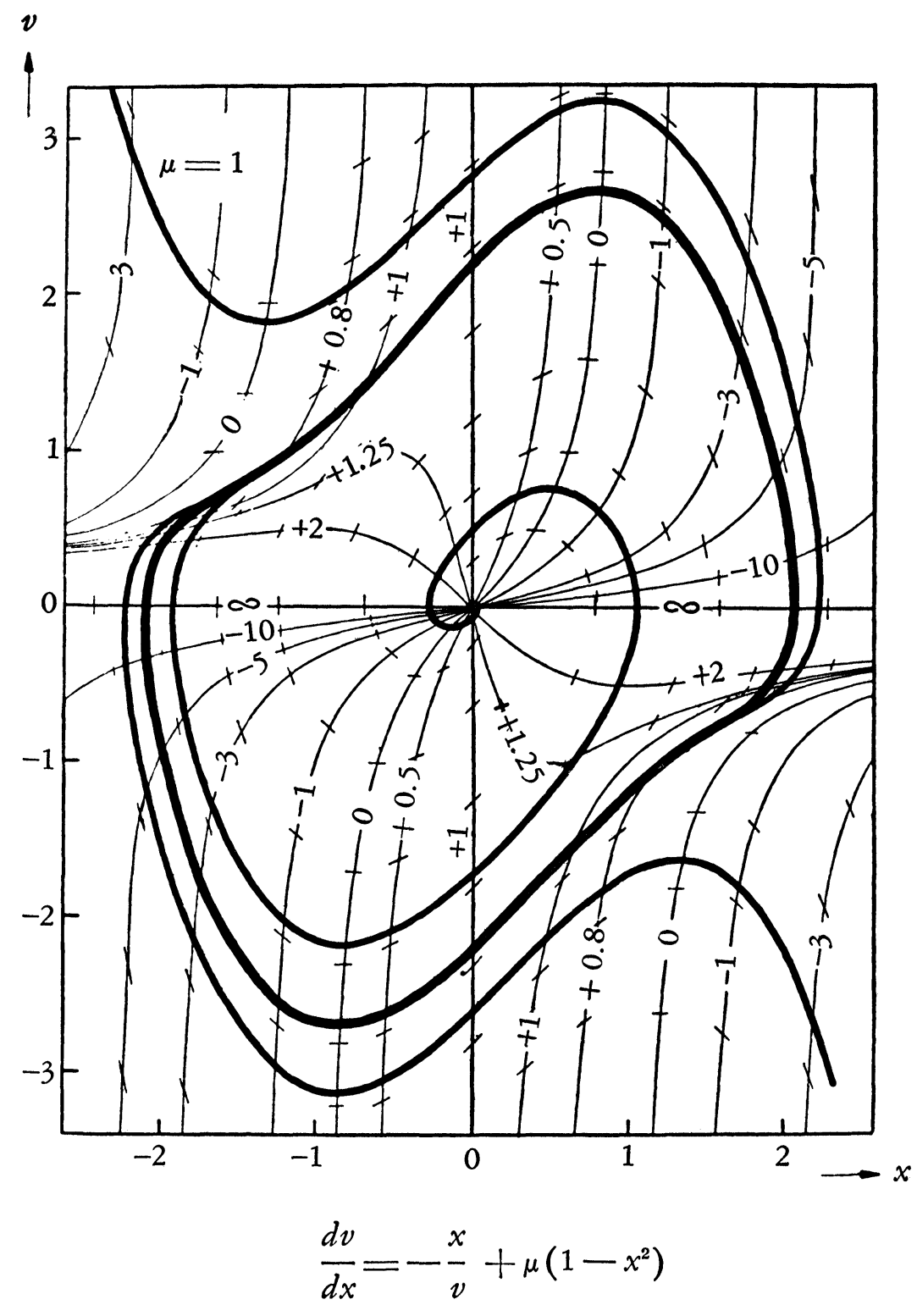

FIG. 3

From B. Van der Pol, Philosophical Magazine, vol. 2 (1926) 
The stability of this type of motion can be investigated by the method of the "equations of variations." Let us assume that

$$
v=\Phi\left(x, x_{0}, v_{0}\right)
$$

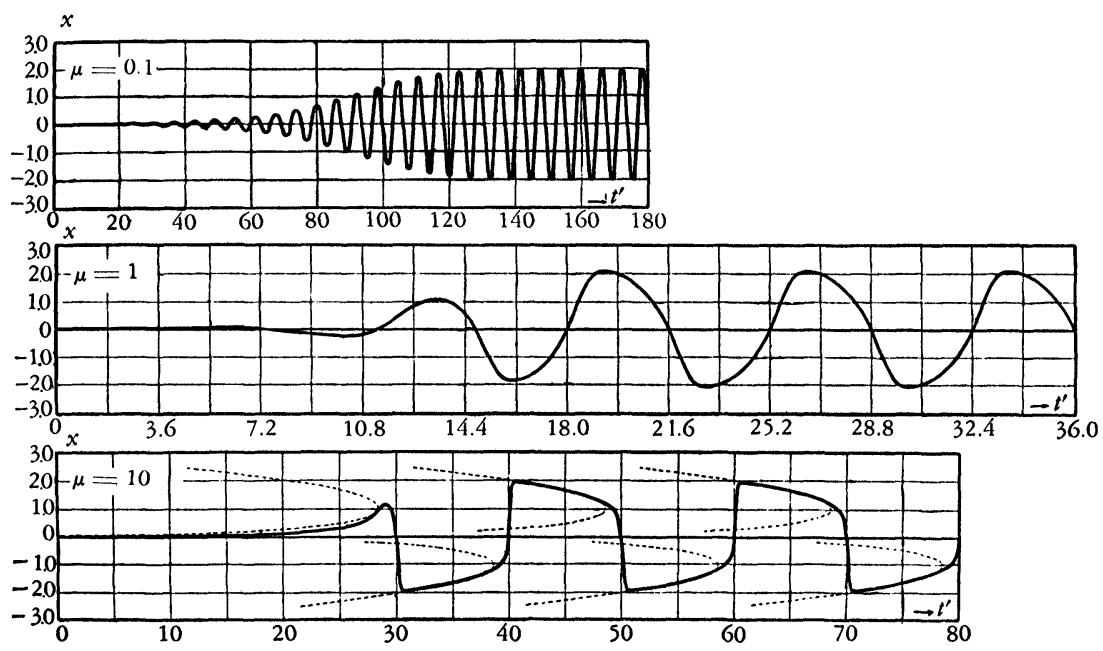

Fig. 4

From B. Van der Pol, Philosophical Magazine, vol. 2 (1926)

is a solution of (2.2) passing through the point $x_{0}, v_{0}$. Then we have

$$
\frac{d}{d x}\left(\frac{\partial v}{\partial x_{0}}\right)=\frac{\partial}{\partial x_{0}}\left(\frac{d v}{d x}\right)=\frac{\partial}{\partial x_{0}}\left[-\omega^{2} \frac{x}{v}+\mu \phi(x)\right]
$$

or

$$
\frac{d}{d x}\left(\frac{\partial v}{\partial x_{0}}\right)=\frac{\omega^{2} x}{v^{2}} \frac{\partial v}{\partial x_{0}}
$$

where $v$ is substituted from equation (2.4) as function of $x, x_{0}$ and $v_{0}$. It is seen that by integrating between the limits $x_{1}$ and $x_{2}$ we have

$$
\left[\log \frac{\partial v}{\partial x_{0}}\right]_{x_{1}}^{x_{2}}=\int_{x_{1}}^{x_{2}} \frac{\omega^{2} x}{v^{2}} d x=-\int_{x_{1}}^{x_{2}} \frac{1}{v} \frac{d v}{d x} d x+\mu \int_{x_{1}}^{x_{2}} \frac{\phi(x)}{v} d x .
$$

Applying this equation to a closed cycle, we obtain

$$
\log \left[\left(\frac{\partial v}{\partial x_{0}}\right)_{2} /\left(\frac{\partial v}{\partial x_{0}}\right)_{1}\right]=\mu \oint \phi(x) d t .
$$


In a similar way we obtain

$$
\log \left[\left(\frac{\partial v}{\partial v_{0}}\right)_{2} /\left(\frac{\partial v}{\partial v_{0}}\right)_{1}\right]=\mu \oint \phi(x) d t
$$

or

$$
\left(\frac{\partial v}{\partial v_{0}}\right)_{2}=\left(\frac{\partial v}{\partial v_{0}}\right)_{1} \operatorname{erp}\{\mu \oint \phi(x) d t\} .
$$

Let us consider now an integral curve in the neighborhood of the closed cycle passing through the point $x_{0}, v_{0}+\delta v_{1}$, and denote the value of $v$ obtained after completing one turn along the closed cycle by $v_{0}+\delta v_{2}$.

Then according to (2.9)

$$
\delta v_{2}=\delta v_{1} \exp \{\mu \oint \phi(x) d t\} .
$$

It is seen that if $\Phi \phi(x) d t>0$ the deviation of a neighboring integral curve from the closed cycle increases; if $\varnothing \phi(x) d t<0$, decreases for each turn. We conclude that the motion corresponding to a periodic

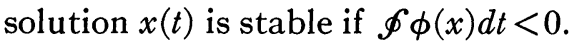

The most important applications of the theory of oscillations of systems with nonlinear damping sketched in this section are in the field of radio. Both the theory and its applications have been treated by several authors. An extensive list of references is given in the paper of Van der Pol mentioned in the Bibliography at the end of this paper [5]. Van der Pol pointed out that in addition to oscillations in mechanical and electrical systems many other periodic phenomena have the character of relaxation oscillations. He found that differential equations similar to those of the relaxation oscillation describe with fair approximation various periodic biological processes, for example, the "struggle for life" of certain animal groups investigated mathematically by A. J. Lotka and Vito Volterra. Van der Pol believes that even periodic business cycles show a certain analogy to the relaxation oscillation of a physical system. The essential condition for such oscillations is negative damping for small deviations and a rather rapidly increasing positive damping for large deviations from the equilibrium position. The psychological response of certain groups of people to changing business conditions shows doubtless some analogy to the behaviour of mechanical systems capable of relaxation oscillations. 
3. Subharmonic resonance. Let us now assume that the function $f(x, \dot{x})$ in equation (1.3) has the form $f(x, \dot{x})=-\mu[\dot{x}+\psi(x)]$; that is, the system has linear damping but the elastic restoring force is a nonlinear function of the deflection. The center of interest is the response of the system to the action of a periodic force; as we mentioned, it was observed that in many cases the oscillations of the natural frequency $\omega$ of the system are excited by a periodic force whose frequency is a multiple of $\omega$. This curious phenomenon is called "subharmonic resonance."

If we consider first the linear system corresponding to $\mu=0$ and assume that a periodic force $a \sin n \omega t$ is applied to the system, the general solution of the equation of motion is

$$
x^{(0)}=c^{(0)} \sin \left(\omega t-\alpha^{(0)}\right)+\frac{-}{\omega^{2}\left(1-\frac{a}{-}-n^{2}\right)} \sin n \omega t .
$$

The first term on the right side represents a free oscillation of the system with an arbitrary amplitude $c^{(0)}$ and phase angle $\alpha^{(0)}$, and the second term a forced oscillation whose frequency is equal to the frequency of the impressed force. If we introduce slight constant damping $[\mu=$ const., $\psi(x)=0]$ the free oscillation dies out and the forced vibration remains with a slightly modified amplitude. Let us now assume that $\psi(x) \neq 0$. Several authors reached the conclusion that in this case the solution consists of higher harmonics with the frequencies $2 n \omega, 3 n \omega, \cdots$. In fact, if one would start from the forced oscillation

$$
\bar{x}^{(0)}=\frac{a}{\omega^{2}\left(1-n^{2}\right)} \sin n \omega t
$$

and determine a next approximation by substituting $\bar{x}^{(0)}$ in $\psi(x)$, it is easily seen that one would obtain forced oscillations whose frequencies are multiples of $n \omega$. However, there is a fallacy in this conclusion because in order to obtain the most general type of periodic motion we have to start with the complete solution (3.1) and put

$$
x=x^{(0)}+\mu x^{(1)}+\mu^{2} x^{(2)}+\cdots .
$$

The function $x^{(0)}$ has two undetermined constants $c^{0}$ and $\alpha^{0}$. They must be chosen in such a way that $x$ is a periodic function of $t$ with the period $T=2 \pi / \omega$. Substituting (3.2) in the differential equation

$$
\ddot{x}+\omega^{2} x=\mu f(x, \dot{x})+a \sin n \omega t
$$

and equating terms of the order $\mu$ on both sides of the equation, we obtain 


$$
\ddot{x}^{(1)}+\omega^{2} x^{(1)}=f\left(x^{(0)}, \dot{x}^{(0)}\right) .
$$

The solution of (3.4) that satisfies the initial conditions $x^{(1)}=\dot{x}^{(1)}=0$ for $t=0$ is given by

$$
x^{(1)}=\int_{0}^{t} f\left[x^{(0)}(\tau), \dot{x}^{(0)}(\tau)\right] \sin \omega(t-\tau) d \tau .
$$

This solution corresponds to a periodic motion with the period $2 \pi / \omega$, if

$$
\begin{aligned}
& x^{(1)}(2 \pi / \omega)=-\int_{0}^{2 \pi} f\left[x^{(0)}(\tau), \dot{x}^{(0)}(\tau)\right] \sin \omega \tau d \tau=0, \\
& \dot{x}^{(1)}(2 \pi / \omega)=\int_{0}^{2 \pi} f\left[x^{(0)}(\tau), \dot{x}^{(0)}(\tau)\right] \cos \omega \tau d \tau=0 .
\end{aligned}
$$

Equations (3.6) are two equations for the undetermined parameters $c^{(0)}$ and $\alpha^{(0)}$. Since $x^{(0)}(t)$ and $\dot{x}^{(0)}(t)$ are periodic functions of $t$ with the period $2 \pi / \omega$, we can expand $f\left[x^{(0)}(t), \dot{x}^{(0)}(t)\right]$ in a Fourier series of the form $\sum_{n} a_{n} \sin n \omega t+\sum_{n} b_{n} \cos n \omega t$. According to equations (3.6) the coefficients $a_{1}$ and $b_{1}$ vanish. Therefore, the solution of equation (3.4) will have the form

$$
\begin{aligned}
x^{(1)}=c^{(1)} \sin \left[\omega t-\alpha^{(1)}\right]+\sum_{n=2}^{\infty} a_{n} & \frac{\sin n \omega t}{\omega^{2}\left(1-n^{2}\right)}+\frac{b_{0}}{\omega^{2}} \\
& +\sum_{n=2}^{\infty} b_{n} \frac{\cos n \omega t}{\omega^{2}\left(1-n^{2}\right)} .
\end{aligned}
$$

Substituting $x=x^{(0)}+\mu x^{(1)}$ and $\dot{x}=\dot{x}^{(0)}+\mu \dot{x}^{(1)}$ into $f(x, \dot{x})$ in equation (3.3), we obtain an equation for $x^{(2)}$ and two equations analogous to equations (3.6). If we neglect higher powers of $\mu$, we have two linear equations for $c^{(1)}$ and $\alpha^{(1)}$. Thus we are able to determine $x$ as function of $t$ to any desired accuracy by successive approximations.

It is seen that the final solution will contain a harmonic oscillation with the frequency $\omega$. This oscillation is called the subharmonic resonance. It has a definite amplitude $c$ and phase $\alpha$, which can be determined by the method of successive approximations described above. Subharmonic resonance occurs if the equations (3.6) have real roots $c^{(0)}$ and $a^{(0)}$. Also it is necessary that the determinant of the linear equations for $c^{(1)}$ and $\alpha^{(1)}$ be different from zero.

The physical meaning of the conditions (3.6) is the following. The expression $\mu f\left(x^{(0)}, \dot{x}^{(0)}\right)$ is an approximate expression for the force resulting from damping and elastic restraint. If the conditions (3.6) are satisfied, that is, the harmonic components of the force with the 
frequency $\omega$ are zero, the work done by this force on the deflection $c^{(0)} \sin \left(\omega t-\alpha^{(0)}\right)$ vanishes.

The problem of subharmonic resonance was treated by a great number of authors. The most important papers are mentioned in the bibliography appended at the end of this lecture. Tuckerman also constructed a simple and ingenious model for the demonstration of subharmonic resonance. ${ }^{2}$ His attention was called to the problem by the fact that certain parts of an airplane can be excited to violent oscillation by an engine running with a number of revolutions much larger than the natural frequency of the oscillating parts.

The method of this section can be applied to forced oscillations of systems with nonlinear damping treated in $\$ 2$. It has been found that also in such systems a force of the frequency $n \omega$ can produce harmonic oscillations of the frequency $\omega$. This procedure in the radio-technique is called "frequency demultiplication." Frequency demultiplication can occur in systems with positive or negative damping for small deflections, that is, also in such systems which are not capable of selfexcited relaxation oscillations.

The mathematical problems outlined in $\S \S 2$ and 3 represent simple cases of problems treated by $\mathrm{H}$. Poincaré in his various researches. The mathematician will readily recognize in the closed curves shown in Figs. 2 and 3 the "cycles limites" studied by Poincaré in his paper, "Sur les courbes définies par une équation différentielle," Oeuvres, vol. 1. The method used in this section is analogous to the methods of the perturbation theory given in the Méthodes nouvelles de la mécanique céleste.

4. Nonlinear problems in the theory of elasticity. Large deflections. In the theory of elasticity usually two fundamental assumptions are made: small deflections and linear stress-strain relations (Hooke's law). Under these assumptions the problem of stress distribution in elastic systems is governed by linear partial differential equations. However, if we give up one or both of these assumptions, we obtain nonlinear equations. We consider in this section the case in which Hooke's law is assumed to be correct, that is, the stresses are linear functions of the strain components, but the deflections produced by external loads are so large that in the expressions for the strain components the squares and products of the deflections and their derivatives cannot be neglected. A classical example of this type is the problem of the "Elastica," that is, the bending and twisting of thin

${ }^{2}$ The author is indebted to Dr. Tuckerman for an extensive list of references and for a film shown during the presentation of this lecture. 
rods. Many of the great mathematicians of the eighteenth and nineteenth century, for example, James and Daniel Bernoulli, Euler, Lagrange and Kirchhoff, were interested in this problem.

Let us consider a simple case: two equal and opposite forces of magnitude $P$, acting along the $x$ axis, are applied to the endpoints of a uniform strut. The endpoints can move freely along the $x$ axis, but are restrained to this axis. The strut can be deflected in the $x, y$

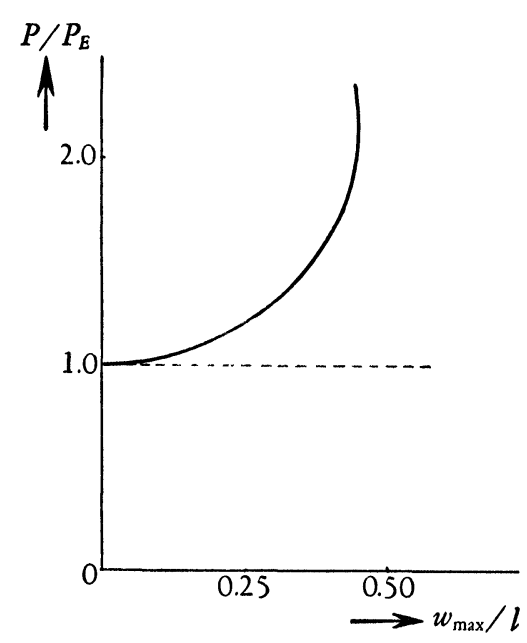

FIG. 5

plane. Then we obtain for the deflection $w$ in the $y$ direction the nonlinear differential equation

$$
\frac{d^{2} w}{d s^{2}}+\frac{P}{B} w\left[1-\left(\frac{d w}{d s}\right)^{2}\right]^{1 / 2}=0
$$

where $s$ is the length of the arc measured along the center line of the strut and $B$ is its bending stiffness. We assume that a positive value of $P$ corresponds to compression in the strut.

For small deflections equation (4.1) becomes

$$
\frac{d^{2} w}{d s^{2}}+\frac{P}{B} w=0 .
$$

If we consider $P$ as parameter in equations (4.1) and (4.2), the eigenvalues of (4.2) which comply with the boundary conditions $w=0$ for $x=0$ and $x=l$ ( $l$ the length of the strut) give us the points of bifurcation of the equilibrium (that is, the branch points of the solu- 
tions). These eigenvalues are equal to $P_{n}=B n^{2} \pi^{2} / l^{2}$, where $n$ is a positive integer. The first characteristic value $(n=1)$ determines the so-called buckling load, or "Euler-load," $P_{E}=\pi^{2} B / l^{2}$ of the strut. The eigenfunction corresponding to $P_{E}$ gives the shape of the buckling column leaving the magnitude of the deflection undetermined. If we are interested in the force-deflection relation beyond the buckling load, we have to integrate equation (4.1), which can easily be done by use of elliptic functions. Fig. 5 shows the ratio between the force $P$ and the buckling load $P_{E}$ as function of the ratio between the deflection $w_{\max }$ of the midpoint and the length $l$ of the beam.

The analogous but much more complicated problem of the finite deflection of plates loaded beyond their buckling limits has great importance for the determination of the strength of certain aircraft structures. The so-called stressed skin (or monocoque) method of construction widely employed for metal airplane wings and fuselages is based on the idea of loading the thin metal skin beyond its buckling limit. In fact the skin in the buckled or "wave" state is able to carry stresses which in many cases are large multiples of the buckling stress. Then the maximum load is determined by the ultimate strength of the buckled skin and the aim of theory is the determination of the stress distribution and the maximum stress occurring in the wave state.

To fix the idea let us assume that a plate of constant thickness $t$, which in the undeflected state is parallel to the $x, y$ plane, is supported freely along two parallel edges, $y= \pm b$, and is subjected to uniformly distributed compressive forces acting normal to its other two edges, $x= \pm a$. The problem is much more complicated than the problem of the Elastica for the following reason: In the case of the deflected thin rod, the resultant of the normal stresses in an arbitrary cross section is determined statically by the end loads. In the case of the deflected plate, the distribution of the resultant stresses acting in the plate depends on the distribution of the normal deflection $w(x, y)$. Let us denote the components of the resultant stresses per unit length of the plate by $\sigma_{x}, \sigma_{y}$ and $\tau_{x y}$. It is known that these stresses are in equilibrium if they can be derived from a function $F(x, y)$ called Airy's function-by the relations

$$
\sigma_{x}=\frac{\partial^{2} F}{\partial y^{2}}, \quad \sigma_{y}=\frac{\partial^{2} F}{\partial x^{2}}, \quad \tau_{x y}=-\frac{\partial^{2} F}{\partial x \partial y} .
$$

If the squares and products of the deflections and strain components are neglected, the function $F(x, y)$ satisfies the linear differential equation 


$$
\Delta \Delta F=0 .
$$

On the other hand if no normal external load is applied to the plate, the normal deflection $w(x, y)$ obeys the equation

$$
B \Delta \Delta w=0
$$

where $B$ is the bending stiffness of the plate per unit length. However, if we take into account the quadratic terms in the deflections and strain components, we obtain the following two simultaneous equations for the functions $F(x, y)$ and $w(x, y)$ :

$$
\begin{aligned}
\Delta \Delta F & =E\left[\left(\frac{\partial^{2} w}{\partial x \partial y}\right)^{2}-\frac{\partial^{2} w}{\partial x^{2}} \frac{\partial^{2} w}{\partial y^{2}}\right], \\
B \Delta \Delta w & =t\left[\frac{\partial^{2} F}{\partial y^{2}} \frac{\partial^{2} w}{\partial x^{2}}+\frac{\partial^{2} F}{\partial x^{2}} \frac{\partial^{2} w}{\partial y^{2}}-2 \frac{\partial^{2} F}{\partial x \partial y} \frac{\partial^{2} w}{\partial x \partial y}\right] .
\end{aligned}
$$

These equations were first given by the present writer in 1910 .

Let us assume that in the undeflected state

$$
\sigma_{x}=-\Pi, \sigma_{y}=\tau_{x y}=0 .
$$

Then substituting these values in the second equation (4.6) we obtain the linearized equation for $w(x, y)$ :

$$
B \Delta \Delta w+\Pi_{t} \frac{\partial^{2} w}{\partial x^{2}}=0 .
$$

This equation has solutions different from $w=0$ which satisfy homogeneous boundary conditions for certain eigenvalues of the parameter $\Pi$.

For example, assuming hinged support at the edges $x= \pm a, y= \pm b$, the eigenvalues will be given by

$$
\Pi=\frac{B}{t} \frac{\pi^{2} a^{2}}{4 m^{2}}\left(\frac{m^{2}}{a^{2}}+\frac{n^{2}}{b^{2}}\right)^{2}
$$

where $m$ and $n$ are integers. These eigenvalues represent the branch points of the equilibrium for the plate. The lowest eigenvalue gives the buckling load $\Pi_{E}$.

If the load $\Pi$ is only slightly higher than this lowest critical value $\Pi_{E}$, the system of equations (4.6) can be solved by successive approximations. However, if the stress applied is many times larger than the "Euler stress" $\Pi_{E}$, this method converges much too slowly for practical purposes. A satisfactory solution of equations (4.6) for large values of $\Pi / \Pi_{E}$ has not as yet been given. The present author 
in a joint paper with E. E. Sechler and L. H. Donnell attacked the problem with a somewhat crude approximation using the notion of the "effective width." The simplifying assumption was made that two narrow strips near the supported edges carry essentially the whole load. The computations of H. L. Cox, M. Yamamoto and K. Kondo, and K. Marguerre are more elaborate; however, they also are not devoid of arbitrary assumptions. Marguerre uses the Rayleigh-Ritz method for the approximate solution of equations (4.6). Since he retains only a few terms of a trigonometric series, his solution if $\Pi / \Pi_{E}$ is very large does not approximate very well the shape of the deflected plate observed in the experiments.

Recently K. Friedrichs and J. Stoker attacked the problem of stress distribution in a circular plate beyond the first buckling, assuming axial symmetry for the deflection. Due to this assumption, the problem is simpler than that of the rectangular plate. They were able to integrate the system of equations (4.6) which in this case are reduced to ordinary differential equations. They investigated the solution for the whole range of values of the ratio $\Pi / \Pi_{E}$, where $\Pi$ is the radial stress per unit area applied on the circumference of the plate and $\Pi_{E}$ is the first critical value of $\Pi$. Below the buckling load, that is, for $\Pi<\Pi_{E}$, the circumferential stress $\sigma_{c}$ is uniformly distributed over the whole plate and is equal to $\Pi$. If $\Pi$ exceeds the buckling stress, $\sigma_{c}$ increases near the edge of the plate and decreases in the center part. For large values of $\Pi / \Pi_{E}, \sigma_{c}$ in the center part becomes negative; it changes from compression to tension, whereas the whole external load is practically carried by a narrow strip near the edge, which is analogous to the effective width introduced by the present writer in the theory of the rectangular plate mentioned above. In this limiting case the method of perturbations can be applied with success since the perturbation term contains the derivative of the highest order. This amounts to an asymptotic integration of the differential equations resulting from equations (4.6). Let us denote the average value of $\sigma_{c}$ by $p$ and the edge stress by $p_{e}$. Then Friedrichs and Stoker obtained the following asymptotic approximation for the relation between $p$ and $p_{e}$

$$
\frac{p}{E(t / a)^{2}}=.73\left[\frac{p_{e}}{E(t / a)^{2}}\right]^{2 / 3}
$$

where $a$ is the radius of the plate.

The same relation with a numerical factor of the same order of magnitude is easily obtained by the method which the present author 
used in the case of rectangular plates. Hence, the analysis of Friedrichs and Stoker can be considered as a verification of the authors' simplifying assumptions.

The theory of large deflections of plates subjected to lateral loads requires the integration of the nonhomogeneous system of equations

$$
\begin{gathered}
\Delta \Delta F=E\left[\left(\frac{\partial^{2} w}{\partial x \partial y}\right)^{2}-\frac{\partial^{2} w}{\partial x^{2}} \frac{\partial^{2} w}{\partial y^{2}}\right], \\
R \Delta \Delta w-t\left[\frac{\partial^{2} F}{\partial y^{2}} \frac{\partial^{2} w}{\partial x^{2}}+\frac{\partial^{2} F}{\partial x^{2}} \frac{\partial^{2} w}{\partial y^{2}}-2 \frac{\partial^{2} F}{\partial x \partial y} \frac{\partial^{2} w}{\partial x \partial y}\right]=p(x, y)
\end{gathered}
$$

where $p(x, y)$ is the lateral load per unit area. This system of equa tions can be solved by successive approximations. Denoting the solution of the nonhomogeneous linear equation

$$
\Delta \Delta w=p / B
$$

by $w_{0}(x, y)$, we substitute this function in the first of the equations (4.10) and determine a first approximation of $F_{1}(x, y)$; then, substituting the derivatives of $F_{1}$ in the second equation (4.10), we calculate the next approximation $w_{1}(x, y)$, and so on. The system of equations (4.10) was solved for certain simple contours and loading conditions by S. Timoshenko and S. Way.

The problem of large deflections of curved rods was treated by several authors. Thus R. Grammel investigated the deformation of a circular ring by uniformly distributed moments along the circular ring axis, which tend to turn the cross sections of the ring around their centers. He found that at a critical value of the moment the ring jumps over into a new equilibrium position.

Another interesting case is the deflection of a flat arch under lateral load where the deflection is of the same order of magnitude as the height of the arch. Let us assume, for example, that the two endpoints of a flat circular arch are pin-joined at two fixed points on the $x$ axis and the arch is loaded by a uniform normal load $p$ per unit length. If $R$ is the radius of curvature of the arch, the uniform normal load produces a compressive force equal to $p R$ and the first bifurcation point of the equilibrium is obtained approximately by Euler's equation for a straight strut:

$$
(p R)_{E}=\pi^{2} \frac{B}{l^{2}}
$$

where $B$ is the bending stiffness, assumed to be constant along the 
length and $l$ is the length of the arch. The nonlinear equation, which takes into account the large deflections, is analogous to the equation of the Elastica mentioned before. However, whereas in the case of the originally straight Elastica the thrust to be applied increases with the deflection, in the case of the originally curved arch, we find that the load $p$ which is necessary to keep the rod in the deflected shape can be smaller than the critical load corresponding to equation (4.12). Thus, as Fig. 5 shows, a straight beam under axial load has only one equilibrium position for values of the parameter $P$, which are smaller than the first eigenvalue $P_{E}$, whereas the flat arch has several possible equilibrium positions for values $p<p_{E}$ as shown in Fig. 6 . Hence the

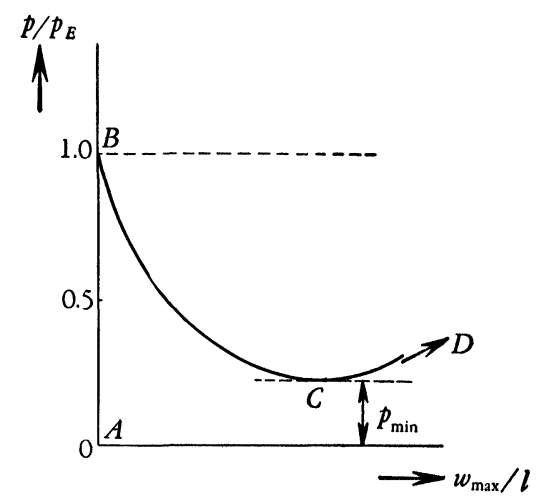

FIG. 6

engineer is more interested in the smallest value of the parameter $p$, which is compatible with the existence of such equilibrium positions than in the value $p_{E}$ corresponding to the branch point of these solutions. To be sure a continuous transition from the unloaded position to such an equilibrium state is only possible if the load $p$ first reaches the critical value $p_{E}$. However, imperfectness of the structure and slight deviations from the uniform load distribution might cause a jump between equilibrium states along $A B$ and $B C$, practically at any value $p$ between $p_{\min }$ and $p_{\theta}$.

It seems to the writer that these considerations are of great importance for the explanation of certain systematic discrepancies found between the breakdown loads of curved shells observed experimentally and computed analytically from the eigenvalues of linearized equations. For example, the differential equation for the small deflections of a thin-walled spherical shell of constant thickness subjected to uniform external pressure was carefully investigated by sev- 
eral authors, for example, R. Zoelly, E. Schwerin, and A. Van der Neut. The critical pressure corresponding to the smallest eigenvalue of the differential equation is found to be equal to

$$
p=\frac{2}{\left[3\left(1-\nu^{2}\right)\right]^{1 / 2}} E\left(\frac{t}{R}\right)^{2}
$$

where $E$ is Young's modulus, $\nu$ Poisson's ratio, $t$ the wall thickness, and $R$ the radius of the spherical shell. The experiments show that the spherical shell collapses under a pressure that is only one-quarter to one-third of this value. Also, the shape of the buckled shell is very different from the wave form predicted by the theoretical eigenfunction that corresponds to the smallest eigenvalue. In a paper published jointly with H. S. Tsien the writer has shown by approximate calculation of the large deflections the existence of more than one equilibrium state at values of the parameter $p$ which are much smaller than the value given by equation (4.13) and are of the order of the values observed experimentally. A similar discrepancy between the prediction of the linear theory and the experimental results in the case of the buckling of cylindrical shells under compression applied parallel to the cylinder axis probably can also be explained by analogous considerations.

Let us finally consider the problem of large deformations of a threedimensional isotropic elastic continuum. This problem, although treated in great detail in the past by several authors, was more or less neglected until interest was stimulated by the possible application of tensor calculus as shown by recent publications of E. Trefftz, F. D. Murnaghan, and M. A. Biot. The first treatment is due to G. Kirchhoff (1852); later J. Boussinesq (1872), M. Brillouin (1891) and J. Finger (1894) were interested in the problem. An excellent presentation of the subject can be found in a review of E. and F. Cosserat (1896). The analysis of all these authors is based on the fundamental assumption that the state of the matter at a point $P$, whose coordinates before the deformation are $x_{i}$, is determined by the deformation of the infinitesimal neighborhood of $P$, that is, by the coefficients of the linear transformation

$$
d \bar{x}_{i}=d\left(x_{i}+\xi_{i}\right)=\frac{\partial \bar{x}_{i}}{\partial x_{k}} d x_{k}
$$

where $x_{i}$ and $\bar{x}_{i}$ are the coordinates of $P$ before and after deformation. The vector whose components are $\bar{x}_{i}-x_{i}=\xi_{i}$ is the displacement vector. The transformation (4.14) has nine coefficients. However, a rigid 
rotation does not produce stresses, and therefore the stresses can only depend on the coefficients of the square of the line element $d \bar{x}_{i}$. We write

$$
d \bar{x}_{i}^{2}=g_{i k} d x_{i} d x_{k}
$$

where

$$
g_{i k}=\alpha_{i l} \alpha_{l k}
$$

and the $\alpha$ 's are the coefficients of the transformation (4.14). In the case of rigid rotation, $g_{i k}=\delta_{i}^{k}$ (that is, $g_{i k}=1$ if $i=k$ and $g_{i k}=0$ if $i \neq k$ ). Hence, we conclude that the six quantities $g_{i k}-\delta_{i}^{k}$ determine the pure deformation of an element. E. and F. Cosserat, and later E. Trefftz, defined these quantities as the components of the strain $\epsilon_{i k}$ and concluded that the stress-strain energy of an isotropic body must be a function of the invariants which can be constructed of the quantities $\epsilon_{i k}$. If the form of the energy function is assumed, the components of the stress tensor are obtained by variation of the energy and application of the principle of virtual displacements.

If the squares and products of the derivatives of the $\xi_{i}$ 's are neglected, $g_{i k}=\delta_{i}^{k}+\partial \xi_{i} / \partial x_{k}+\partial \xi_{k} / \partial x_{i}$. Hence, in this case the transformation (4.14) can be split into two parts:

$$
d \xi_{i}=\frac{1}{2}\left(\frac{\partial \xi_{i}}{\partial x_{k}}+\frac{\partial \xi_{k}}{\partial x_{i}}\right) d x_{k}+\frac{1}{2}\left(\frac{\partial \xi_{i}}{\partial x_{k}}-\frac{\partial \xi_{k}}{\partial x_{i}}\right) d x_{k} .
$$

The first term represents an infinitesimal pure deformation, the second term an infinitesimal rigid rotation. The quantities $\epsilon_{i k}$ $=\frac{1}{2}\left(\partial \xi_{i} / \partial x_{k}+\partial \xi_{k} / \partial x_{i}\right)$ are known as components of the strain tensor. If Hooke's law is valid, the stress-strain energy is a homogeneous quadratic form of the $\epsilon_{i k}$ 's, and the components of the stress tensor are derivatives of this quadratic form with respect to the $\epsilon_{i k}$ 's.

If higher terms in the derivatives of the $\xi_{i}$ 's are taken into account, two difficulties arise:

(a) The separation of the pure deformation from the rotation and the definition of the strain tensor becomes somewhat arbitrary.

(b) One must differentiate between stresses referred to the surface elements of the undeformed and deformed system.

M. A. Biot suggested the following definition of the rotation and the strain tensor: The linear transformation

$$
\bar{x}_{i}=x_{i}+\frac{\partial \xi_{i}}{\partial x_{k}} x_{k}
$$


can be replaced by two successively applied linear transformations, where the first one:

$$
x_{i}^{\prime}=\beta_{i k} x_{k}
$$

is an orthogonal transformation representing a finite rigid rotation and the coefficients of the second one:

$$
\bar{x}_{i}=\gamma_{i k} x_{k}^{\prime}
$$

constitute a symmetrical matrix, that is, $\gamma_{i k}=\gamma_{k i}$. Then the first transformation defines in a unique way the rotation and the second one the pure deformation. The strain tensor is defined by

$$
\epsilon_{i k}=\frac{1}{2}\left(\gamma_{i k}-\delta_{i}^{k}\right)
$$

We notice that the $\gamma_{i k}$ 's are equal to the $g_{i k}$ 's only in the first approximation; they differ if higher terms in the derivatives of the $\xi_{i}$ 's are taken into account.

F. D. Murnaghan's definition of the strain tensor is based on the inverse transformation

$$
d x_{i}=\frac{\partial x_{i}}{\partial \bar{x}_{k}} d \bar{x}_{k}
$$

If we express the square of the line element $d s_{i}$ by $d \bar{s}_{i}$, we obtain an expression of the form

$$
d s_{i}^{2}=h_{i k} d \bar{x}_{i} d \bar{x}_{k}
$$

Then we can consider

$$
\epsilon_{i k}=\frac{1}{2}\left(\delta_{i}^{k}-h_{i k}\right)
$$

as the components of the strain tensor.

Using different conceptions for the strain tensor, Hooke's generalized law appears in different forms in the publications mentioned. Fortunately the question is of academic rather than practical importance. With the exception of a few materials with large elastic extension, such as rubber, the strain components are very small within the limit of elasticity, and the usual first approximation is entirely satisfactory. However, if at least one dimension of the body is small compared to the others, as in the case of plates, shells, and beams, it is possible that some of the nine derivatives $\partial \xi_{i} / \partial x_{k}$ are large, although the six components of the strain tensor are small. This takes place in some of the problems mentioned before in this section. It appears that 
the most important task of the general transformation theory of finitc deformations should be a systematic and exact verification of the differential equations for large deflection of beams, plates and shells. The present deduction of these equations is based on intuitive assumptions rather than on an exact application of the general equations of elasticity for a three-dimensional continuum.

5. Stress distribution in plastic and noncoherent masses. Let us drop now the assumption that the material obeys Hooke's law. Two types of nonlinear strain-stress relations have practical importance. First, in some materials, although there is a unique and reversible relation between stress and strain, the linear law does not approximate this relation with a sufficient accuracy. Second, we find that beyond a certain stress limit the deformation is no longer purely elastic; that is, after removing the load, we obtain a so-called permanent set. The nonreversible part of the deformation is known as plastic deformation or plastic flow; the stress limit beyond which plastic flow is obtained is called the yield point. In general if plastic flow takes place the yield point is raised, that is, additional plastic flow can only be produced by further increase of the load. This effect is called cold hardening of the material. Stress distribution in bodies with nonlinear elastic behaviour and in materials undergoing cold hardening were treated analytically only incidentally. However, the limiting case in which cold hardening can be neglected is readily accessible to analytical treatment and has been the subject of quite a large number of ingenious investigations.

A material that is capable of plastic deformation without cold hardening is called perfectly plastic. The experiments show that the yield condition of a perfectly plastic material is given by a constant difference between the largest and smallest principal stress. This condition must be satisfied in the whole domain in which plastic flow takes place. Let us restrict ourselves to two-dimensional problems, assuming that the material flows in planes parallel to the $x, y$ plane. In this case the value of the third principal stress, which is parallel to the $z$ axis, necessarily lies between the values of the two other principal stresses. If we derive the stresses $\sigma_{x}, \sigma_{y}, \tau_{x y}$ from the stress function $F(x, y)$ by means of the relations

$$
\sigma_{x}=\frac{\partial^{2} F}{\partial y^{2}}=t, \quad \sigma_{y}=\frac{\partial^{2} F}{\partial x^{2}}=r, \quad \sigma_{x y}=-\frac{\partial^{2} F}{\partial x \partial y}=-s,
$$

the square of the difference of the principal stresses $\sigma_{1}$ and $\sigma_{2}$ is equal to 


$$
\left(\sigma_{1}-\sigma_{2}\right)^{2}=(r+t)^{2}-4\left(r t-s^{2}\right) .
$$

Hence, the yield condition can be written in the form

$$
(r+t)^{2}-4\left(r t-s^{2}\right)=K^{2},
$$

where $K$ is a characteristic constant for the material.

Equation (5.3) is a nonlinear partial differential equation of the
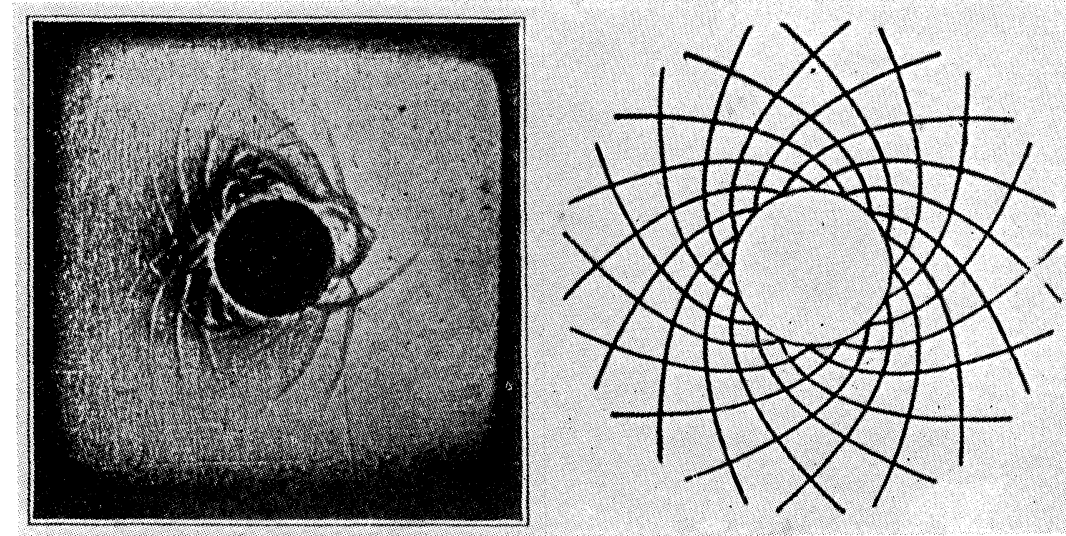

FIG. 7

From A. Nadai, Plasticity

second order of the hyperbolic type. The equation of the characteristics is

$$
(r-t)\left[\left(\frac{d y}{d x}\right)^{2}-1\right]-4 s \frac{d y}{d x}=0
$$

or

$$
2 \frac{d y}{d x} /\left[1-\left(\frac{d y}{d x}\right)^{2}\right]=\frac{r-t}{2 s}
$$

Let us denote in an arbitrary point the angle between the tangents to the characteristic curves passing through this point and the positive $x$ axis by $\theta_{1}$ and $\theta_{2}$; then according to equation (5.5) $\theta_{1}$ and $\theta_{2}$ satisfy the equation

$$
\tan 2 \theta=\frac{r-t}{2 s}
$$

Now the angles $\phi_{1}$ and $\phi_{2}$ between the directions of the principal 
stresses $\sigma_{1}$ and $\sigma_{2}$ and the $x$ axis are the roots of the equation

$$
\tan 2 \phi=\frac{2 \tau_{x y}}{\sigma_{x}-\sigma_{y}}=\frac{2 s}{r-t} .
$$

From (5.6) and (5.7) we obtain $\tan 2 \theta \tan 2 \phi=1$. The curves which follow the direction of the principal stresses are known as isostatics. Hence, the characteristics are a system of orthogonal curves that in-
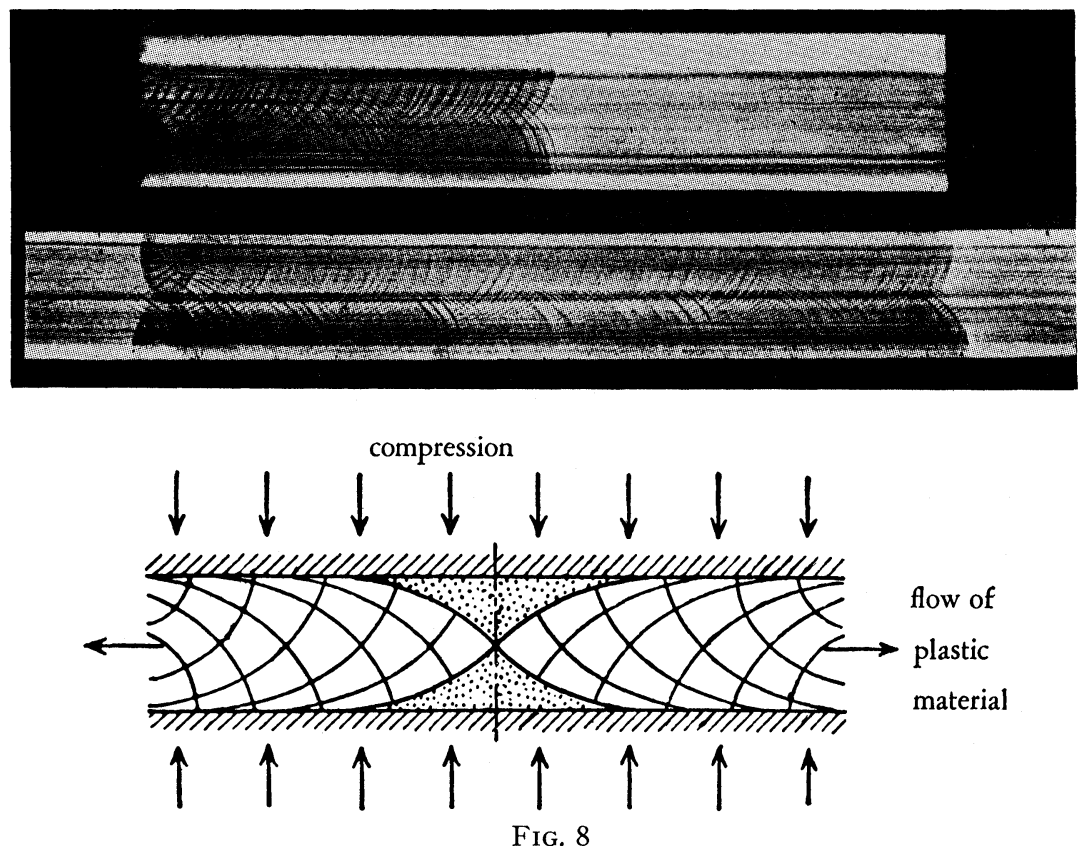

From A. Nadai, Plasticity

tersect the isostatics at $45^{\circ}$ and are tangential to the direction of the maximum shearing stress. Now the plastic flow consists essentially of a gliding along the planes in which the maximum shearing stresses occur. Therefore the characteristics are the traces of the gliding surfaces in the $x, y$ plane. Sometimes they are visible on the surface of the body subjected to plastic flow without any artifice (Fig. 7); in other cases they can be brought out by etching (Fig. 8).

The geometrical properties of the characteristics given by equation (5.5) and the integration of the differential equation (5.3) by means of Riemann's method were studied by H. Hencky, L. Prandtl, C. Carathéodory and E. Schmidt. Let us introduce the parameters of 
the characteristics $\alpha$ and $\beta$ as independent variables, the average normal stess $p=\frac{1}{2}(r+t)$, and the coefficients $A$ and $B$ of the line element

$$
d s^{2}=A d \alpha^{2}+B d \beta^{2}
$$

as unknown functions. Then we obtain the equations

$$
\frac{\partial^{2} p}{\partial \alpha \partial \beta}=\frac{K}{2}
$$

and

$$
\frac{1}{B} \frac{\partial A}{\partial \beta}=f(\alpha), \quad \frac{1}{A} \frac{\partial B}{\partial \alpha}=g(\beta)
$$

where $f(\alpha)$ and $g(\beta)$ are arbitrary functions of the parameters $\alpha$ and $\beta$. To integrate the equations (5.9) and (5.10) we start from two given characteristic curves $\alpha=0$ and $\beta=0$ and choose the values of $A(\alpha, 0)$ and $B(0, \beta)$ along these curves, putting, for example, $A(\alpha, 0)=B(0, \beta)$ $=1$. Since the characteristic curves are orthogonal,

$$
-\frac{1}{B} \frac{\partial A}{\partial \beta}=\frac{\partial \theta}{\partial \alpha}
$$

where $\theta$ is the angle between the $x$ axis and the tangent of a curve $\beta=$ const., the tangent being drawn in the direction of increasing $\alpha$; similarly

$$
\frac{1}{A} \frac{\partial B}{\partial \alpha}=\frac{\partial \theta}{\partial \beta}
$$

Hence, the functions $f(\alpha)$ and $g(\beta)$ are known and the system of equations (5.10) can be integrated by Riemann's method. If the network of characteristic curves is so determined, equation (5.9) gives the value of $p$. Then the magnitudes of the principal stresses can be calculated from $\frac{1}{2}\left(\sigma_{1}+\sigma_{2}\right)=p$ and $\left|\sigma_{1}-\sigma_{2}\right|=K$. The stresses are directed at every point parallel to the bisectors of the characteristic curves.

The stress distribution in a mass of sand which is in the limiting state of equilibrium, that is, just about to collapse, can be determined in a similar manner. If the sand is under a large external load and the influence of its own weight can be neglected, the stress function $F(x, y)$ satisfies the equation

$$
(r+t)^{2}-\frac{4}{1-f^{2}}\left(r t-s^{2}\right)=0
$$

where $f$ is the coefficient of friction between the sand particles. This equation is identical to the fundamental equation of the theory of 
earth pressure developed by Rankine and can be applied to various problems in soil mechanics. However, in many applications gravity has to be taken into account. The differential equation of the stress function in this case is no longer homogeneous, but contains also terms which are linear in $r, t$ and $s$. Fortunately, in a few cases of practical importance the partial differential equation can be reduced to an ordinary equation; such problems have been treated by several authors.

Both in the theory of plastic and noncoherent masses, so far we have assumed that the stress distribution is two-dimensional. If we try to extend the theory to the three-dimensional case, the difficulty arises that the gliding conditions (5.3) and (5.11) involve only two principal stresses leaving the third principal stress undetermined. There are two ways of attacking the problem. First, one can consider the equilibrium of the whole system as a variation problem: the potential energy of the system has to be a minimum under the restriction that the yield condition is not violated at any point. Hence, we have to solve a variation problem, where the extremals satisfy an inequality. The solution of this variation problem leads to the equation (5.3) or (5.11) for the largest and smallest principal stresses and to an additional equation for the median principal stress.

The second method of dealing with the problems of three-dimensional stress distribution is to introduce certain empirical stress and rate of strain relations for the plastic deformation. One can assume, for example, proportionality between the stress tensor and the derivative of the strain tensor with respect to time. This assumption replaces Hooke's law and furnishes the necessary compatibility equations for the determination of the third principal stress. The same two methods can be applied to the practically quite important case in which one part of the body is in the elastic and another part in the plastic state. Both methods furnish a sufficient number of boundary conditions for the surface separating the elastic and plastic domains. However, this surface is not a priori given and, therefore, the solution of the problem is in general very difficult. It has been worked out only in a few geometrically simple cases.

6. Hydrodynamics of ideal incompressible fluids. In this and the following sections we will consider nonlinear problems occurring in fluid mechanics. In $\$ \$ 6$ to 8 we assume a nonviscous and incompressible fluid; in $\$ 9$ we shall take into account viscosity, and in $\$ 10 \mathrm{com}$ pressibility.

The velocity distribution in an ideal incompressible fluid can be determined by the so-called Eulerian equations of motion. Denoting 
the components of the velocity by $u_{i}$, the density of the fluid by $\rho$, the pressure by $p$ and the components of the volume forces per unit mass by $F_{i}$, we have three equations for the dynamic equilibrium:

$$
\frac{\partial u_{i}}{\partial t}+u_{j} \frac{\partial u_{i}}{\partial x_{j}}=-\frac{1}{\rho} \frac{\partial p}{\partial x_{i}}+F_{i},
$$

and the kinematic equation of continuity:

$$
\frac{\partial u_{i}}{\partial x_{i}}=0 .
$$

The equations (6.1) are nonlinear in the $u_{i}$ 's. However, if we assume that the flow is without vorticity, that is, the $u_{i}$ 's are derivatives of a velocity potential $\phi$, and that the forces $F_{i}$ are derivatives of a force potential $V$, the three equations (6.1) are satisfied by their first integral

$$
\frac{\partial \phi}{\partial t}+\frac{1}{2} \frac{\partial \phi}{\partial x_{i}} \frac{\partial \phi}{\partial x_{i}}+\frac{p}{\rho}-V=\text { const., }
$$

and the equation of continuity becomes

$$
\Delta \phi=0 .
$$

Hence, in the case of irrotational motion the problem of determining the velocity distribution is reduced to finding solutions of Laplace's equation (6.4) that satisfy certain boundary conditions. These boundary conditions are in certain cases linear. For example, if the flow takes place between solid boundaries, the component of the velocity normal to these boundaries vanishes, and, therefore, $\partial \phi / \partial n$ $=0$. Hence, in this case the problem of finding the velocity distribution is reduced to a well known linear boundary problem of the potential theory.

If the fluid is bounded by a free surface, that is, by another fluid of negligible specific gravity which is at rest, the boundary condition has to express that the pressure $p$ is constant along the free boundary. Hence, we have from equation (6.3)

$$
\frac{\partial \phi}{\partial t}+\frac{1}{2} \frac{\partial \phi}{\partial x_{i}} \frac{\partial \phi}{\partial x_{i}}-V=\text { const. }
$$

Assuming that gravity is the only force acting on the fluid, we substitute $V=-g x_{3}$, where $g$ is the acceleration of gravity directed parallel to the negative $x_{3}$ axis, and obtain 


$$
\frac{\partial \phi}{\partial t}+\frac{1}{2} \frac{\partial \phi}{\partial x_{i}} \frac{\partial \phi}{\partial x_{i}}+g x_{3}=\text { const. }
$$

The difficulty of solving flow problems with free boundary arises not only from the nonlinear character of the boundary condition (6.5), but also from the fact that the boundary itself is unknown. Threedimensional problems of this type have been solved only in a few cases; hence, we shall restrict ourselves to two-dimensional flow in the $x_{1}, x_{3}$ plane. We also assume that the flow is stationary, that is, $\phi$ is independent of time and therefore $\partial \phi / \partial t=0$.

Replacing the coordinates $x_{1}$ and $x_{3}$ by $x$ and $y$, we have at a free surface the boundary condition

$$
\frac{1}{2}\left[\left(\frac{\partial \phi}{\partial x}\right)^{2}+\left(\frac{\partial \phi}{\partial y}\right)^{2}\right]+g y=\text { const. }
$$

Let us introduce the complex variable $z=x+i y$ and two analytical functions of $z$, namely, $f=\phi+i \psi$, where $\phi$ is the velocity potential and $\psi$ the stream function, and $\omega=\theta+i \log q$, where $\theta$ is the angle between the velocity vector and the $x$ axis, and $q$ the magnitude of the velocity vector. The components of the velocity of flow in the $x$ and $y$ direction are equal to $u=\partial \phi / \partial x$ and $v=\partial \phi / \partial y$ respectively. Hence, $u-i v=d f / d z$ and, therefore, $\omega=i \log (u-i v)$. Then $\theta=\tan ^{-1} v / u$ and

$$
\log q=\log |u-i v|=\frac{1}{2} \log \left[\left(\frac{\partial \phi}{\partial x}\right)^{2}+\left(\frac{\partial \phi}{\partial y}\right)^{2}\right]
$$

are harmonic functions of $x$ and $y$ and also of $\phi$ and $\psi$. Putting $\tau=\log q$, we write the boundary condition (6.6) in the form

$$
\frac{1}{2} e^{2 \tau}+g y=\text { const. }
$$

or, by differentiating with respect to the arc along the free boundary,

$$
e^{2 \tau} \frac{\partial \tau}{\partial s}+g \frac{\partial y}{\partial s}=0
$$

Taking into account that the free boundary is a streamline ( $\psi=$ const.)

$$
\frac{\partial \tau}{\partial s}=\frac{\partial \tau}{\partial \phi} q=\frac{\partial \tau}{\partial \phi} e^{\tau} .
$$

On the other hand, $\partial y / \partial s=\sin \theta$ and, therefore, we obtain from (6.8)

$$
\frac{1}{3} \frac{\partial}{\partial \phi}\left(e^{3 r}\right)=-g \sin \theta \text {. }
$$


We now consider $\phi$ and $\psi$ as independent variables; then we have to satisfy the boundary (6.9) condition along a horizontal straight line $\psi=$ const. in the $\phi, \psi$ plane, where $\Delta \theta=0$ and $\Delta \tau=0$. We remember that $\theta$ and $\tau$ are the real and imaginary parts of an analytical function of $\phi+i \psi$.

The nonlinear boundary condition (6.9) can be linearized in the following two cases:

(a) If the effect of gravity is negligible and the fixed boundaries are plane surfaces, then we obtain for the free boundary, from (6.9),

$$
\frac{\partial \tau}{\partial \phi}=0
$$

The fixed boundaries being plane surfaces, their traces in the $x, y$ plane are straight lines, that is, along such boundaries $\theta$ is constant, and we have therefore

$$
\frac{\partial \theta}{\partial \phi}=0 .
$$

This case was treated in great detail by G. Kirchhoff, H. Helmholtz, Lord Rayleigh and others, and has been applied to many practical problems such as the vena contracta, the flow of water over a weir, the air resistance of an inclined flat plate, the planing of a flat plate over a water surface, and so on.

(b) If gravity is taken into account and the motion is only slightly different from a uniform parallel flow with the velocity $U$, we can assume that $\theta$ is small and $\tau=T+u / U$, where $T=\log U$. Neglecting the squares and products of $\theta, u$ and $v$, we have from equation (6.9)

$$
U^{2} \frac{\partial u}{\partial \phi}=-g \theta
$$

or

$$
U^{3} \frac{\partial \theta}{\partial \psi}+g \theta=0
$$

Let us assume that $\psi=0$ corresponds to the free surface, $\psi=-\infty$ to the infinite depth of the water mass. We also assume that $\theta, u$ and $v$ disappear at infinite depth. Then

$$
\theta=\theta_{0} e^{-\lambda \psi} \sin \lambda \phi
$$

satisfies the equation $\Delta \theta=0$ and the boundary condition for $\psi=-\infty$. 
We still have to satisfy the boundary condition (6.13) for $\psi=0$. Substituting (6.14) into equation (6.13), we have

$$
-U^{3} \lambda+g=0
$$

and, therefore,

$$
\theta=\theta_{0} e^{-g \psi / U^{3}} \sin g \phi / U^{3} .
$$

Let us denote the equation of the free surface by $\eta=\eta(x)$. With the

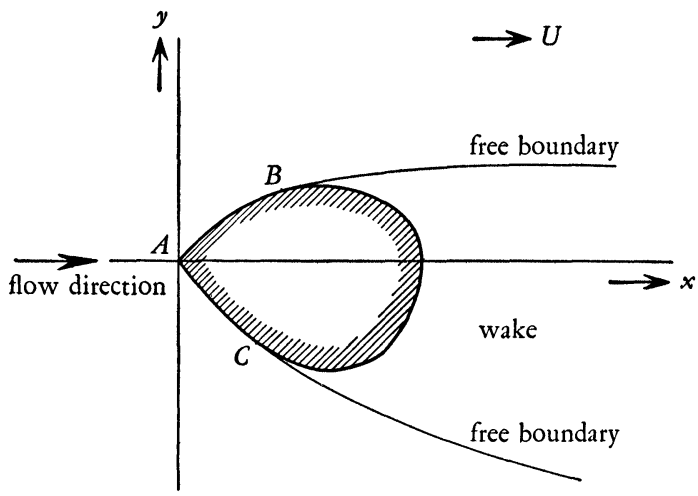

FIG. 9

approximation used here we can replace $\phi$ by $U x$ and $d \eta / d x$ by $\theta$. Hence, we obtain

$$
\eta=\eta_{0} \cos \frac{x g}{U^{2}}
$$

where $\eta_{0}=-\theta_{0} U^{2} / g$.

If we consider the flow relative to a coordinate system moving from right to left with the velocity $U$, the surface (6.17) represents a wave progressing with the velocity $U$ on the surface of a water mass which is at rest at infinite depth. The wave length $L$ is equal to $2 \pi U^{2} / g$.

The solution of waves of small amplitude outlined here is the classical solution of Airy and Stokes. In the next two sections we drop the restrictions that made the linearization of the boundary conditions possible and consider the resulting nonlinear problems.

7. Fluid jets. T. Levi-Civita was the first to extend the theory of Kirchhoff and Helmholtz to jets bounded by curved survaces. In a paper published in the Rendiconti del Circolo Matematico di Palermo in 1907 , he treated the problem of the wake behind a blunt cylindrical body (Fig. 9). Let us assume that the fluid follows the curved surfaces 
$A B$ and $A C$, and that behind the body a dead fluid region, the socalled wake, is bounded by free surfaces which extend from $B$ and $C$ to infinity. If we consider $\phi$ and $\psi$ as independent variables, and $\theta$ and $\tau=\log q$ as two harmonic functions of $\phi$ and $\psi$, the boundary condition for the free surfaces is $\tau=$ const. To find the boundary condition for a fixed curved surface it is convenient to use so-called natural equations. For example, we can express the inclination $\theta$ as function of the length of arc $s$. Then the curvature $d \theta / d s$ is also a known function of $\theta$, say $d \theta / d s=k K(\theta)$, where $k$ is a numerical parameter. Now the trace of the fixed surface is a streamline, and, therefore,

$$
\frac{\partial \theta}{\partial s}=\frac{\partial \theta}{\partial \phi} \frac{\partial \phi}{\partial s}=e^{\tau} \frac{\partial \theta}{\partial \phi} .
$$

Hence, we obtain the boundary condition

$$
\frac{\partial \theta}{\partial \phi}=k e^{-\tau} K(\theta) .
$$

It is seen that for plane surfaces $K(\theta)=0$. Then we have the special case treated by Kirchhoff, Helmholtz and Lord Rayleigh and equation (7.1) is reduced to equation (6.11).

Levi-Civita, by use of convenient transformations of the variables, reduced the problem to the following:

To find an analytic function $\theta+i \tau$ of a complex variable $\zeta=\xi+i \eta$, which is zero for $\zeta=0$, regular in the half of the unit circle above the real axis, and whose real and imaginary parts satisfy the following boundary conditions:

(a) on the circumference of the unit half circle

$$
\frac{d \theta}{d \sigma}=k f(\sigma) e^{-\tau} K(\theta)
$$

where $\sigma$ is the argument of $\zeta$ on the unit circle and $f(\sigma)$ is a known function of $\sigma$.

(b) on the diameter along the real axis $\tau=0$.

The problem can be solved by successive approximations or by expansion in a series of powers of the parameter $k$.

The theory of Levi-Civita was further developed by S. Brodetzky, C. Schmieden, S. Bergmann, V. Valcovici, R. Thirry, L. Rosenhead and others. An interesting aspect of this theory is that the point of separation, that is, the point at which the free boundary begins, is undetermined between certain limiting values. These limiting values are determined by the conditions that the free boundaries shall not 
intersect either the fixed boundaries or each other. The question of existence and uniqueness of the solution for a given separation point was discussed by A. Weinstein, G. Hamel, H. Weyl and K. Friedrichs.

If we take gravity into account and the fixed surfaces are curved, the boundary conditions are nonlinear both at the free and the fixed boundaries. The theory of such "heavy" jets has many important practical applications in the design of weirs, spillways, and so on. A spillway (Fig. 10) is a steep channel at the downstream face of a dam,

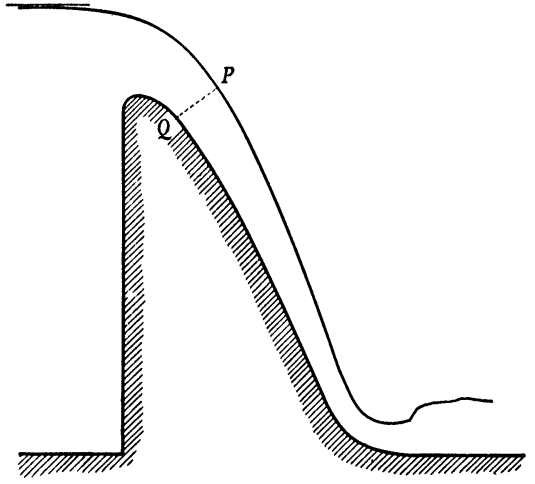

FIG. 10

designed for the smooth discharge of large quantities of water. The engineer has to design the spillway in such a way that the pressure does not sink below the atmospheric pressure at any point on the surface where the water is in contact with the spillway. If we compare the pressure at the points $P$ and $Q$ - that is, on the opposite sides of a cross section normal to the average flow direction-we see that the height difference between $P$ and $Q$ causes the pressure to be higher at $Q$ whereas the centrifugal force causes the pressure to be higher at $P$. A correct design requires an approximate balance between these two opposite effects. If the pressure at $Q$ is sub-atmospheric, air enters between the jet and the wall and might cause an unstable jumping of the jet between two possible flow patterns; one of them is a jet with two free boundaries and the other one a jet with one free and one fixed boundary. Cases are known in which the instability of the jet has caused such violent oscillations of the air that when the spillway was in use windows were rattled within a radius of several miles around the damsite. Many engineers have "grappled" with the mathematical solution of the spillway problem; however, the mathematical 
analysis has not furnished sufficiently dependable information, so that spillway design is based mostly on the results of model experiments.

8. Waves with finite amplitude. The theory of waves with infinitestimally small amplitude sketched in $\S 6$ is due to G. B. Airy (1845) and G. G. Stokes (1847). Stokes himself, and later Lord Rayleigh, T. H. Havelock, A. G. M. Michell and several other authors gave further approximations to the exact shape of a wave of finite amplitude progressing without change (so-called wave of permanent type). However, the systematic treatment of the problem, including proofs for the existence and convergence of solutions, has been given only recently by T. Levi-Civita and his collaborators, D. J. Struik and A. Weinstein.

The analytical problem involved in the theory of waves of finite amplitude progressing on the surface of a water mass of infinite depth can be formulated in the following way:

To find all functions $\theta+i \tau$ of a complex variable $\zeta=\rho e^{i \sigma}$ that are holomorphic in the interior of the unit circle $|\zeta|=1$, have the value zero at $\zeta=0$, and satisfy at the circumference of the unit circle the condition

$$
\frac{d \tau}{d \sigma}=p e^{-3 \tau} \sin \theta
$$

where $p=g L / 2 \pi U^{2}$ is a positive undetermined parameter. The symbols $\theta, g, L$ and $U$ have the meaning given in $\S 6$ and $\tau=\log g / U$. The variable $\zeta$ is connected with the complex potential function $f=\phi+i \psi$ used in $\$ 6$ by the relation

$$
\zeta=e^{2 \pi i f / U L} .
$$

If $\tau$ and $\theta$ are small, we have approximately $d t / d \sigma=p \theta$ or

$$
\rho \frac{\partial \theta}{\partial \rho}=p \theta .
$$

The problem is in this case a linear eigenvalue problem; the solution is

$$
\theta=\theta_{0} \rho^{p} \sin p\left(\sigma-\sigma_{0}\right)
$$

where $p$ is an integer and $\theta_{0}$ and $\sigma_{0}$ are arbitrary constants. However, it can be shown that physically the solutions corresponding to different integral values of $p$ are all identical. If we choose, for example, $p=n$, this only means that we consider a group of $n$ identical waves 
with the wave length $L$ as one wave of the wave length $n L$. Hence, it is sufficient to consider the case $p=1$, which gives the relation

$$
U^{2}=\frac{g L}{2 \pi}
$$

between the velocity of propagation $U$ and the wave length $L$, in

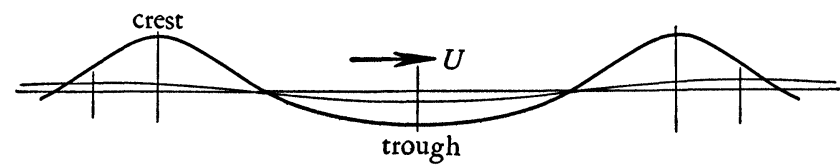

FIG. 11

accordance with the result given in $\$ 6$. The factor in equation (8.4) and, therefore, the height of the waves in this approximation remain undetermined.

Levi-Civita has shown that if $p<1$ but $1-p$ is sufficiently small, a certain wave shape of definite height/length ratio corresponds to every value of $p$. He also obtained convergent series for the calculation of the wave shape. He uses as a first approximation the solution of the linear problem for $p=1$, namely,

$$
\theta+i \tau=-i \mu \zeta=-i \mu \rho \cos \sigma+\mu \rho \sin \sigma
$$

where $\mu$ is a real constant. Then the solution which satisfies the exact boundary condition (8.1) can be written in the form

$$
\theta+i \tau=\sum_{n=1}^{\infty} \omega_{n}(\zeta) \mu^{n}
$$

where the $\omega_{n}(\zeta)$ 's are polynomials of the $n$th degree in $\zeta$. Levi-Civita calculated numerically the polynomials $\omega_{n}(\zeta)$ up to $n=5$. Fig. 11 shows an example of the wave shape resulting from his computations compared with the shape of an infinitesimally small wave of the same velocity of propagation. The limiting smallest value of $p$ and the corresponding shape of the highest wave has not as yet been exactlydetermined. G. Stokes believed that the highest wave has a sharp edge at the crest with a vertex angle of $120^{\circ}$.

D. J. Struik extended the investigations of Levi-Civita to waves of finite height in canals of finite depth. A. Weinstein investigated the interesting problem of the so-called "solitary wave," that is, a single elevation of the water level travelling with constant velocity and pre- 
serving the same shape along a canal of finite depth. This phenomenon was studied as early as 1844 by J. Scott Russel, who observed disturbances of this type generated by the motion of large barges in

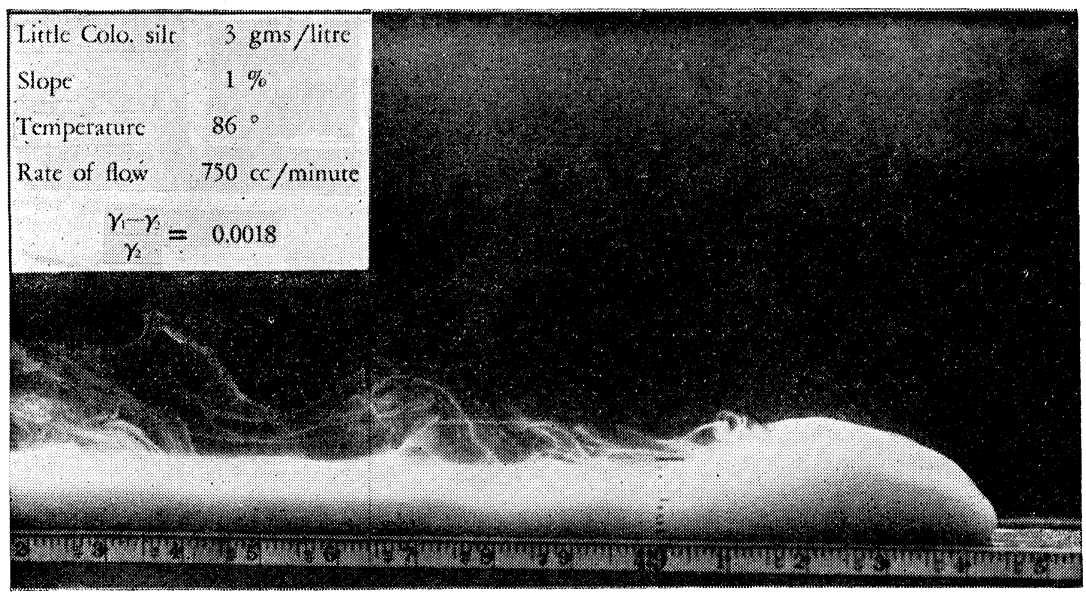

FIG. 12

Courtesy of H. S. Bell, Soil

Conservation Service

relatively narrow canals. He followed them several miles on horseback and measured in this way their velocity of propagation.

Nonlinear problems of fluid motion in rivers and artificial channels were treated in the "hydraulic manner" by J. Boussinesq. The "hydraulic manner" of dealing with flow problems consists essentially in replacing the partial differential equations for the velocity distribution by an ordinary differential equation for the mean velocity averaged over a cross section. By combining this dynamical equation with the equation of continuity, one obtains an ordinary nonlinear differential equation for the height of the water level. A great number of problems treated in this way can be found, for example, in P. Forchheimer's book Hydraulik, Teubner, 1930.

Interesting nonlinear problems related analytically and physically to the problem of surface waves arise in the analysis of the flow in stratified fluid media. The stability of heavy fluids of different densities flowing one above the other with different velocities was investigated by means of the method of small oscillations by H. Helmholtz; $\mathrm{V}$. Bjerknes considered the small perturbations occurring along plane 
surfaces of discontinuity between fluids of different densities for several combinations of density and velocity distribution. However, the exact shape of separating surfaces with large curvature has not yet been thoroughly investigated.
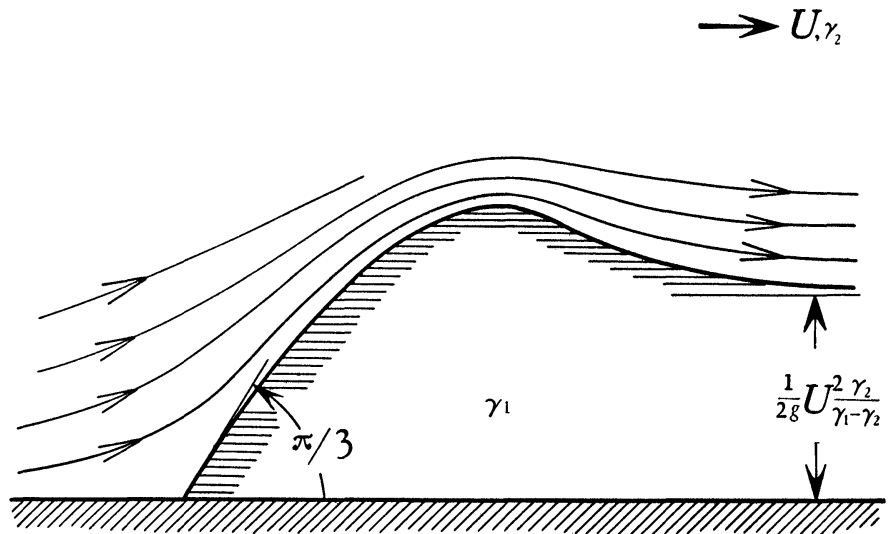

FIG. 13

A case of this type is, for example, the intrusion of a heavy fluid mass moving with constant velocity into a mass of lighter fluid, as illustrated by the meteorological phenomenon of a progressing cold front or by the intrusion of muddy water into a reservoir filled with clear water, or salt water into a mass of fresh water. Fig. 12 shows the shape of the surface separating muddy and clear water. The front of muddy water progresses with approximately constant speed and keeps its permanent shape. Hence, if we consider the flow relative to a coordinate system moving with the heavier fluid, the latter can be assumed to be at rest and the lighter fluid to be in stationary motion (Fig. 13). The pressure $p$ must be equal on both sides of the discontinuity surface. Then, if the specific gravity of the heavier fluid is $\gamma_{1}$ and that of the lighter fluid $\gamma_{2}$, the equilibrium condition for the heavy fluid yields the condition $p / \gamma_{1}+y=$ const., and Bernoulli's equation applied to the lighter fluid leads to $p / \gamma_{2}+y+q^{2} /(2 g)=$ const., where $y$ is the height measured from the bottom and $q$ is the velocity of the lighter fluid along the discontinuity surface. Taking into account that, at the intersection of the discontinuity surface and the bottom, $y=0$ and $q=0$, we obtain the condition for the discontinuity surface: 


$$
\frac{q^{2}}{2 g}-\frac{\gamma_{1}-\gamma_{2}}{\gamma_{2}} y=0 .
$$

It is seen that (8.8) is a nonlinear boundary condition of the type of equation (6.6). The main interest is directed toward the determination of the shape of the discontinuity surface. We can show-using a reasoning similar to that employed by G. G. Stokes for the determination of the steepest slope occurring in waves of finite height - that if frictional effects are neglected the slope of the discontinuity surface at the intersection with the bottom must be equal to $\pi / 3$. The complex velocity potential for the flow of the lighter fluid in the neighborhood of the intersection $z=x+i y=0$ has the form

$$
f=C z^{\alpha}
$$

where $\alpha$ and $C$ are real, positive constants. Hence, the magnitude of the velocity is given by $|d f / d z|=C \alpha\left|z^{\alpha-1}\right|$, and, therefore, $q$ is proportional to $y^{\alpha-1}$ and $q^{2}$ to $y^{2(\alpha-1)}$. Since, according to equation (8.8), $q^{2}$ is proportional to $y$, we must have $2 \alpha-2=1$ or $\alpha=\frac{3}{2}$. Hence, $f=C z^{3 / 2}$ or $z=(f / C)^{2 / 3}$. Since the streamline $\psi=0$ consists of the bottom line and the trace of the discontinuity surface, and $f=0$ corresponds to the point of intersection, the angle between the two branches of the streamline $\psi=0$ is equal to the change in the value of the imaginary part of $\log z=\frac{2}{3} \log (f / C)$, when we pass from $\phi<0$ to $\phi>0$, that is, equal to $2 \pi / 3=120^{\circ}$.

In an actual experiment (Fig. 12) the friction on the bottom causes a certain distortion of the discontinuity surface; however, if this friction is diminished by introducing a thin fluid layer at rest under the progressing muddy water, the slope obtained experimentally is in fair agreement with the calculated value. It can also be shown analytically that the front of the heavy fluid must have a so-called head, whose peak is considerably higher than the mean thickness of the heavy layer, which is equal to

$$
y=\frac{\gamma_{2}}{\gamma_{1}-\gamma_{2}} \frac{U^{2}}{2 g},
$$

where $U$ is the velocity of progression of the front. However, the shape of the discontinuity surface has not yet been determined; it is not known whether a mathematical analysis would lead to a surface approaching the horizontal level asymptotically or to waves with decreasing or constant amplitudes.

Also, the nonlinear boundary problems resulting from the analysis of the motion of planing surfaces have not yet been attacked seri- 
ously. The shape of the free water surface behind a planing surface has been determined by $\mathrm{H}$. Wagner, but he neglected the effect of gravity. The investigators who have taken into account gravity ${ }^{3}$ assumed small elevations and depressions of the water surface. The gap between these two first approximations ought to be filled by further investigations.

9. Viscous fluids. The dynamical equations for the flow of a viscous incompressible fluid have the form:

$$
\frac{\partial u_{i}}{\partial t}+u_{k} \frac{\partial u_{i}}{\partial x_{k}}=-\frac{1}{\rho} \frac{\partial p}{\partial x_{i}}+\nu \Delta u_{i}
$$

where $i=1,2,3$, and $\nu$ is the kinematic viscosity of the fluid; the continuity equation is, in accordance with equation (6.2)

$$
\frac{\partial u_{i}}{\partial x_{i}}=0 .
$$

Equations (9.1) are known as the equations of Navier and Stokes.

In the case of viscous fluids we have to assume that at fixed boundaries not only the normal but also the tangential components of the velocity vanish. The equations (9.1) and (9.2) are to be integrated with these boundary conditions.

There are a few cases in which this system of nonlinear equations can be exactly solved by known functions:

(a) In some cases the solutions which satisfy the linear part of the equation make the nonlinear terms vanish identically. Thus the problem is reduced to the integration of a system of linear equations. For example, if only one component of the velocity, for example, the component $u_{1}$, is different from zero, from equation (9.2) it follows that $u_{1}$ is a function of $x_{2}$ and $x_{3}$ only. Let us consider stationary flow; then we have to conclude from equations (9.1) that $\partial p / \partial x_{2}=\partial p / \partial x_{3}=0$ and

$$
\nu\left(\frac{\partial^{2} u_{1}}{\partial x_{2}^{2}}+\frac{\partial^{2} u_{1}}{\partial x_{3}^{2}}\right)=\frac{1}{\rho} \frac{d p}{d x_{1}}=\text { const. }
$$

The solution of this equation furnishes the velocity distribution in a cylindrical pipe. The boundary condition is $u_{1}=0$ at the wall.

(b) Certain solutions of the equations for irrotational flow of nonviscous fluids lead to exact solutions of the system of equations (9.1) and (9.2) if we assume that the streamlines remain unaltered, while

${ }^{3}$ Cf., for example, Lamb's Hydrodynamics, pp. 375-383. 
the magnitude of the velocity changes. A typical flow of this type is the two-dimensional radial flow between two divergent or convergent walls. Writing equations (9.1) and (9.2) in polar coordinates and denoting the radial velocity by $u_{r}$, we obtain the dynamical equations for stationary flow in the form

$$
\begin{gathered}
u_{r} \frac{\partial u_{r}}{\partial r}=-\frac{1}{\rho} \frac{\partial p}{\partial r_{1}}+\nu\left(\frac{\partial^{2}}{\partial r^{2}}+\frac{1}{r} \frac{\partial}{\partial r}+\frac{1}{r^{2}} \frac{\partial^{2}}{\partial \theta^{2}}-\frac{1}{r^{2}}\right) u_{r}, \\
0=-\frac{1}{\rho} \frac{1}{r} \frac{\partial p}{\partial \theta}+\frac{2 \nu}{r^{2}} \frac{\partial u_{r}}{\partial \theta}
\end{gathered}
$$

while the continuity equation is solved by

$$
u_{r} r=f(\theta) .
$$

We introduce $f(\theta)$ as the unknown function, substituting $u_{r}=f(\theta) / r$ in equations (9.4), and eliminate the pressure $p$ from these two equations. Then we obtain for $f(\theta)$ the ordinary differential equation:

$$
f^{\prime \prime}+4 f+\frac{1}{\nu} f^{2}=\text { const. }
$$

which can be integrated by use of elliptic functions. The boundary conditions are $u_{r}=0$ for $\theta= \pm \alpha$, where $\alpha$ is half of the angle of convergence or divergence. The interesting feature of this problem is that if the flow is convergent, equation (9.6) has only one solution which satisfies the boundary conditions at the walls; however, if the flow is divergent and the total amount of fluid flowing through a cross section $Q=r \int_{-\alpha}^{+\alpha} u_{r} d \theta$ exceeds a certain value, the solution is no longer unique. For example, for the same value of $Q$, we may obtain a symmetrical and two asymmetrical solutions. The symmetrical solution represents a flow in which the fluid shoots with high velocity through the center of the diverging channel and back flow takes place at both sides of the jet. The two asymmetrical solutions represent flow patterns with positive velocity near one wall and backflow in the neighborhood of the other wall. For very large values of $Q$ the sign of the velocity changes many times between $\theta=-\alpha$ and $\theta=\alpha$. This solution was found and discussed by the present writer in collaboration with one of his Ph.D. candidates in 1913. The young man was killed at the beginning of the first world war and the investigation remained unpublished. Shortly afterward the same solution was found by G. Hamel as a special case of a wider class of exact solutions of the Navier-Stokes equations.

Methods for approximate integration of the differential equations 
(9.1) are known for two limiting cases: namely, for very large and very small values of the parameter $\nu$.

(A) If $\nu$ is large, we obtain a first approximation by neglecting the quadratic terms, so that we have

$$
\frac{\partial u_{i}}{\partial t}=-\frac{1}{\rho} \frac{\partial p}{\partial x_{i}}+\nu \Delta u_{i} .
$$

Eliminating $p$ and using equation (9.2), we obtain three linear differential equations for $u_{1}, u_{2}$ and $u_{3}$. These equations were integrated for certain geometrically simple cases by G. G. Stokes. The second approximation should be obtained by substituting the first approximation in the quadratic terms. It is difficult to find a satisfactory justification for this method of approximation. As a matter of fact, it was shown by $\mathrm{C}$. W. Oseen that in the case of a solid moving in a fluid, the solution of (9.7) represents a first approximation only in the neighborhood of the solid, no matter how large the parameter $\nu$ may be.

(B) From an engineering point of view the limiting case of small viscosity is more important. This topic is known as the theory of the laminar boundary layer in the engineering literature. The basic mathematical idea of this theory is the approximate solution of the hydrodynamic equations by asymptotic integration. L. Prandtl's fundamental paper On the theory of fluids with small viscosity was presented at the First International Congress for Mathematics in 1904 in Heidelberg and drew very little attention from the mathematicians attending this congress who were much more concerned with the problems of George Cantor's Mengenlehre and other subjects of pure mathematics, on which interest was focused in those days, than in the speculations of a young professor of applied mechanics, whose presentation perhaps was not very clear from a purely mathematical point of view. Several years passed before the method was correctly understood. Nowadays it is widely used in the theory of skin friction and many other hydrodynamical, aerodynamical and meteorological phenomena in which inertia forces and viscous forces are of the same order of magnitude.

Let us consider the following simple problem: An incompressible viscous fluid flows along a flat plate that extends along the positive $x$ axis from $x=0$ to $x=\infty$ (Fig. 14). (We shall use the coordinates $x, y, z$ instead of $x_{1}, x_{2}, x_{3}$.) No other boundaries exist; the velocity of flow at $x=-\infty$ is directed parallel to the $x$ axis and is equal to $U$. We assume two-dimensional stationary motion and introduce the stream function $\psi(x, y)$ defined by the relations 


$$
u=\frac{\partial \psi}{\partial y_{1}}, \quad v=-\frac{\partial \psi}{\partial x}
$$

which satisfy identically the continuity equation. Then the dynamical equations yield the following differential equation of fourth order for $\psi$ :

$$
\frac{\partial \psi}{\partial y} \frac{\partial \Delta \psi}{\partial x}-\frac{\partial \psi}{\partial x} \frac{\partial \Delta \psi}{\partial y}=\nu \Delta \Delta \psi .
$$

If $\nu=0$, the presence of the plate has no influence on the motion and

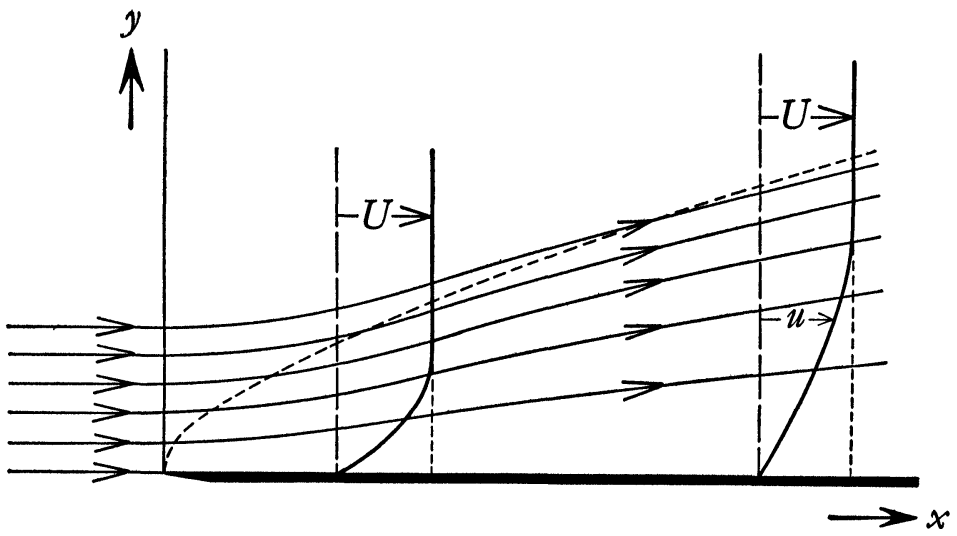

FIG. 14

we have $u=U$ or $\psi_{0}=U y$; the only boundary condition at $y=0$ is $\nu=0$. However, if $\nu \neq 0$, no matter how small $\nu$ may be, we have the additional boundary condition $u=0$ for $y=0$.

To obtain an approximate solution for small values of $\nu$, we use the method of perturbations, starting from the solution for $\nu=0$. We notice that the perturbation term contains higher derivatives of $\psi$ than the differential equation (9.8) with $\nu=0$. It is known that in such a case we have to change the independent variable by a substitution which contains the parameter $\nu$ so that we write

$$
\psi=U y+\nu^{\alpha} \psi_{1}(x, \eta)
$$

where $\eta=y \nu^{\beta}$ and $\alpha$ is positive. The exponents $\alpha$ and $\beta$ are to be determined in such a way that $u=\partial \psi / \partial y$ is of the order of $U$ and the largest terms on the left and right sides of equation (9.8) are of the same order. The first condition leads to $\alpha+\beta=0$. Substituting the 
expression (9.9) into equation (9.8) and putting $\beta=-\alpha$, we find that the largest terms on the left side are

$$
\frac{\partial \psi}{\partial y} \frac{\partial^{3} \psi}{\partial x \partial y^{2}}=\nu^{-\alpha}\left(U+\frac{\partial \psi_{1}}{\partial \eta}\right) \frac{\partial^{3} \psi_{1}}{\partial x \partial \eta^{2}}
$$

and

$$
-\frac{\partial \psi}{\partial x} \frac{\partial^{3} \psi}{\partial y^{3}}=-\nu^{-\alpha} \frac{\partial \psi_{1}}{\partial x} \frac{\partial^{3} \psi_{1}}{\partial \eta^{3}} ;
$$

the largest term on the right side is $\nu \partial^{4} \psi / \partial y^{4}=\nu^{1-3 \alpha} \partial^{4} \psi_{1} / \partial \eta^{4}$. These terms are of the same order if $\alpha=\frac{1}{2}$. Hence equation (9.9) becomes

$$
\psi=\nu^{1 / 2} U \eta+\nu^{1 / 2} \psi_{1}(x, \eta)
$$

where $\eta=y / \nu^{1 / 2}$.

With this substitution, equation (9.8) takes the form

$$
\left(U+\frac{\partial \psi_{1}}{\partial \eta}\right) \frac{\partial^{3} \psi_{1}}{\partial x \partial \eta^{2}}-\frac{\partial \psi_{1}}{\partial x} \frac{\partial^{3} \psi_{1}}{\partial \eta^{3}}=\frac{\partial^{4} \psi_{1}}{\partial \eta^{4}} .
$$

This equation can be integrated once with respect to $\eta$ :

$$
\left(U+\frac{\partial \psi_{1}}{\partial \eta}\right) \frac{\partial^{2} \psi_{1}}{\partial x \partial \eta}-\frac{\partial \psi_{1}}{\partial x} \frac{\partial^{2} \psi_{1}}{\partial \eta^{2}}=\frac{\partial^{3} \psi_{1}}{\partial \eta^{3}}+f(x) .
$$

Since $\partial \psi_{1} / \partial \eta$ and $\partial^{2} \psi_{1} / \partial \eta^{2}$ are zero for $\eta=\infty$, the arbitrary function $f(x)$ must be zero. We notice that the substitution $\psi_{1}(x, \eta)=x^{1 / 2} f\left(\eta / x^{1 / 2}\right)$ reduces equation (9.12) to an ordinary differential equation for the function $f$ where $\zeta=\eta / x^{1 / 2}$. In fact, we obtain

$$
-\frac{1}{2}(U \zeta+f) f^{\prime \prime}=f^{\prime \prime \prime} \text {. }
$$

This differential equation has been integrated numerically for the boundary conditions $f=0, f^{\prime}=-U$ at $\zeta=0$ and $f^{\prime}=0$ at $\zeta=\infty$, by H. Blasius (1908) and more accurately by C. Töpfer (1912). The region near the wall where $f$ or the stream function of the perturbation, $\psi_{1}(x, y)$, is materially different from zero is called the "boundary layer." The order of magnitude of the extension of this region normal to the wall is $(\nu x / U)^{1 / 2}$; we say in engineering language that we have a boundary layer of the thickness $\delta$, where $\delta$ is of the order of $(\nu x / U)^{1 / 2}$. To fix ideas, $\delta$ can be defined by the relation $\nu^{1 / 2} \int_{\infty}^{0} \partial \psi_{1} / \partial \eta d \eta=U \delta$. This value of $\delta$ is called the displacement thickness, and is given by $\delta=1.72 /(\nu x / U)^{1 / 2}$. The velocity distribution in the boundary layer is given by $u=U+f^{\prime}$. The calculated values of $u / U$ are plotted in 
Fig. 15 and compared with experiment. The solution of equation (9.13) determines also the skin friction, that is, the shearing stress

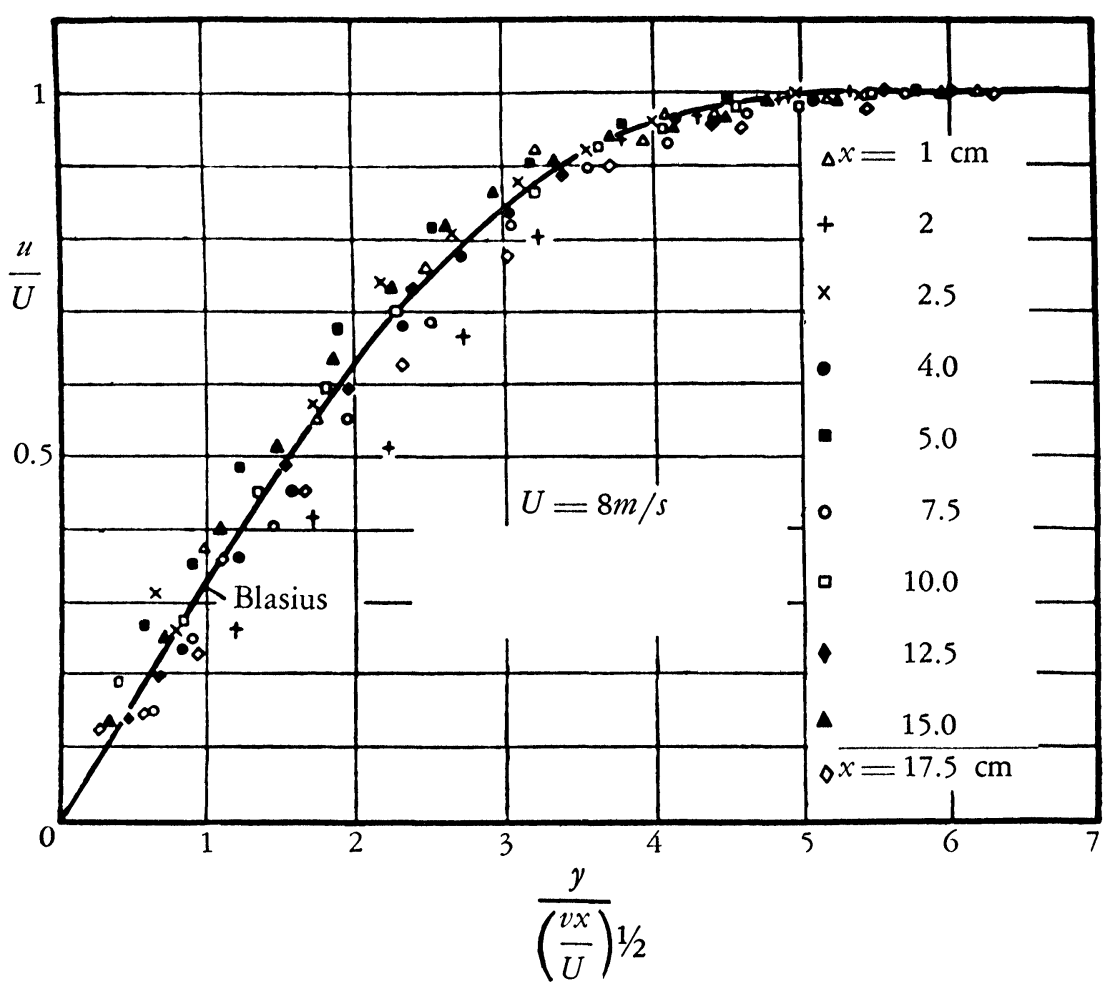

Fig. 15

From Hansen, Aachen Abhandlungen, vol. 8

at the surface; its value per unit area of the plate is variable with $x$, that is, with the distance from the leading edge of the plate. At a distance $x$, we have

$$
\tau=\rho \nu\left(\frac{\partial u}{\partial y}\right)_{y=0}=0.66414 \frac{\rho U^{2}}{2} \frac{1}{(U x / \nu)^{1 / 2}} .
$$

The dimensionless quantity $U x / \nu$ is called the Reynolds number of the plate extending from $x=0$ to $x=x$.

We have carried through this calculation in the manner of the calculus of perturbations. Prandtl considered the hydrodynamic equations in the form given by (9.1) and (9.2) and determined the relative 
orders of magnitude of the terms in these equations, assuming that the velocity component $u$ changes from its value $U$ to zero in a small range near the wall. Denoting the order of magnitude of this range by $\delta$, he concludes that $\delta$ must be of the order $\nu^{1 / 2}$ and $\partial u / \partial y, \partial^{2} u / \partial y^{2}$ of the order $1 / \nu^{1 / 2}, 1 / \nu$ respectively while $\partial u / \partial x$ is of standard order. Then from equations (9.1) it follows that the derivative of the pressure with respect to a coordinate normal to the wall is of the order $\nu^{1 / 2}$, hence negligible in the approximation used in this theory. In this way we obtain, for example, in the case of uniform flow along a flat plate, the simplified equations:

$$
u \frac{\partial u}{\partial x}+v \frac{\partial u}{\partial y}=\nu \frac{\partial^{2} u}{\partial y^{2}}, \quad \frac{\partial u}{\partial x}+\frac{\partial v}{\partial y}=0 .
$$

The boundary conditions are $u=v=0$ at the wall and $u=U$ at $y=\infty$, where $U$ is the velocity "outside of the boundary layer," that is, the velocity resulting for $y=0$ from the solution of the equation $\Delta \psi_{0}=0$ for nonviscous fluids.

If we have two-dimensional flow along a wall but the velocity $U$ is not constant, equations (9.15) take the form:

$$
u \frac{\partial u}{\partial x}+v \frac{\partial u}{\partial y}=U \frac{d U}{d x}+\nu \frac{\partial^{2} u}{\partial y^{2}}, \quad \frac{\partial u}{\partial x}+\frac{\partial v}{\partial y}=0 .
$$

Prandtl has shown that these equations can also be applied to the flow along a curved surface, provided the lines $x=$ const. are normal to the surface, $x$ is the length of arc measured at the surface and $y$ is the normal distance from the surface. Equations (9.16) furnish a fair approximation if $K \delta$ and $(d K / d x) \delta^{2}$ are sufficiently small, $K$ the curvature of the wall and $\delta$ the thickness of the boundary layer.

The integration of the system of equations (9.16) has great importance for many applications. Various attempts have been made to obtain approximative solutions. An excellent review of the methods applied is given in Modern Developments in Fluid Dynamics, edited by $\mathrm{S}$. Goldstein. Here only a short account will be given:

(a) S. Goldstein showed that equations $(9.16)$ can be reduced to an ordinary differential equation if $U(x)$ has the form const. $x^{n}$ or const. $e^{\lambda x}$. The case $n=0$ leads to the case treated above; $n=1$ that is, $U=$ const. $x$ corresponds to a boundary layer starting from a stagnation point of the main flow. This problem has been treated by Blasius, Hiemenz, Howarth and Bickley. The equation for arbitrary $n$ has been studied by Falkner and Skan and by Hartree. Special cases were discussed by R. Mills. 
(b) Let us assume that $U(x)$ is given in the form of a power series

$$
U=\beta_{1} x+\beta_{2} x^{2}+\beta_{3} x^{3}+\cdots .
$$

Then we put $u=\partial \psi / \partial y, v=-\partial \psi / \partial x$ and

$$
\psi=F_{1} x+F_{2} x^{2}+F_{3} x^{3}+\cdots .
$$

By substituting (9.17) and (9.18) in equations (9.16) and equating the terms with the same power of $x$, we obtain systems of ordinary differential equations for the functions $F_{1}, F_{2}, \cdots$ of $y$. This method was used by Hiemenz, Howarth, Bickley and others.

(c) We might start from an "initial velocity profile" at $x=0$, assuming, for example,

$$
u(0, y)=\alpha_{1} y+\alpha_{2} \frac{y^{2}}{2 !}+\alpha_{3} \frac{y^{3}}{3 !}+\cdots \cdot
$$

and calculate the "next profile," that is, $u(\Delta x, y)$. However, in general, that is, for arbitrary values of $\alpha_{1}, \alpha_{2}, \cdots$, we obtain a singularity at $x=y=0$. If the function $U(d U / d x)$ has the form

$$
U \frac{d U}{d x}=\gamma_{1} x+\gamma_{2} x^{2}+\cdots,
$$

the function $\psi(x, y)$ is regular only if certain relations between the coefficients $\alpha_{1}, \alpha_{2}, \cdots$ and $\gamma_{1}, \gamma_{2}, \cdots$ are satisfied. Hence, the application of a step-by-step method progressing in the $x$-direction is rather difficult.

(d) A method of obtaining approximate values of the thickness of the boundary layer and the skin friction as function of $x$ has been proposed by the present writer and worked out in detail by Pohlhausen. By integrating the first equation of (9.16) between $y=0$ and an arbitrary fixed value of $y$ and using the condition of continuity, we obtain

$$
\frac{d}{d x} \int_{0}^{y} u^{2} d y+[u v]_{0}^{y}=\nu\left[\frac{\partial u}{\partial y}\right]_{0}^{y}+\frac{d}{d x} \int_{0}^{y} \frac{U^{2}}{2} d y .
$$

Taking into account the second equation (9.16), the second term on the left side of (9.20) becomes $-U(d / d x) \int_{0}^{y} u d x$ and we obtain

$$
\frac{d}{d x} \int_{0}^{y}\left(U^{2}-u^{2}\right) d y-U \frac{d}{d x} \int_{0}^{y}(U-u) d y=-\nu\left[\frac{\partial u}{d y}\right]_{0}^{y}
$$

Extending the integral to a value $y=\delta$, where $U-u$ is negligible, that 
is, over the whole cross section of the boundary layer, we can assume that $\partial u / \partial y=0$ for $y=\delta$ and we have

$$
\frac{d}{d x} \int_{0}^{\delta}\left(U^{2}-u^{2}\right) d y-U \frac{d}{d x} \int_{0}^{\delta}(U-u) d y=\nu\left(\frac{\partial u}{\partial y}\right)_{0} .
$$

This equation, given first by the present writer, is an expression for the conservation of the momentum of the fluid involved in the boundary layer. Let us assume that the velocity distribution between $y=0$ and $y=\delta$ can be expressed by a polynomial of the form

$$
U-u=U\left[1-a \frac{y}{\delta}+b\left(\frac{y}{\delta}\right)^{2}+c\left(\frac{y}{\delta}\right)^{3}\right]
$$

where $a, b$ and $c$ are functions of $x$. Then from $U-u=0$ and $\partial u / \partial y=0$ at $y=\delta$ follows: $1-a+b+c=0$ and $-a+2 b+3 c=0$. For $y=0$ we have, from the first equation of $(9.16), \nu\left(\partial^{2} u / \partial y^{2}\right)+U(d U / d x)=0$; hence

$$
\frac{2 b \nu}{\delta^{2}}+\frac{d U}{d x}=0
$$

It is seen that $a, b$ and $c$ are expressed by $\delta$ and $d U / d x$. Therefore, if we substitute (9.24) into equation (9.23), we obtain an ordinary nonlinear differential equation of first order for $\delta$.

This method was applied with success in many cases and gave fair approximations for engineering purposes. It has the disadvantage that it is difficult to estimate the degree of approximation. If the actual shape of the velocity distribution profile is very different from the assumed polynomial, the result can be misleading.

(e) R. von Mises suggested a transformation of coordinates for equation (9.16), introducing $x$ and $\psi$ as independent variables and $Z=U^{2}-u^{2}$ as the unknown function. Then $u(\partial / \partial x+(v / u) \partial / \partial y)$ has to be replaced by $u \partial / \partial x$ and $\partial / \partial y$ by $u \partial / \partial \psi$. We obtain in this way

$$
\frac{\partial Z}{\partial x}=\nu u \frac{\partial^{2} Z}{\partial \psi^{2}}
$$

where $u=\left(U^{2}-Z^{2}\right)^{1 / 2}$ and $U$ is a given function of $x$. If we compare this equation with equation (9.12) it is seen that its order is reduced from 3 to 2 . However, an additional quadrature is necessary to obtain the velocity as function of $x$ and $y$. von Mises suggested a step-bystep method to solve equation (9.25). The difficulty caused by a singularity at $x=\psi=0$ mentioned under item (c) occurs also in this case.

(f) If we introduce instead of $x$ the variable $\phi=\int_{0}^{x} U d x$ (that is, the 
value of the potential function obtained by solving the equation $\Delta \psi_{0}=0$ for the nonviscous case), equation (9.25) can be written in the form

$$
\frac{\partial Z}{\partial \phi}=\nu \frac{u}{U} \frac{\partial^{2} Z}{\partial \psi^{2}} .
$$

In the outer portion of the boundary layer, $u / U$ is not very different from unity. Then we find that $Z$ satisfies approximately the heat conduction equation:

$$
\frac{\partial Z}{\partial \phi}=\nu \frac{\partial^{2} Z}{\partial \psi^{2}} .
$$

The boundary condition is $Z=[U(x)]^{2}$ for $\psi=\infty$. The solution satisfying equation (9.27) and this boundary condition might be called the "outer solution." We shall now calculate the "inner solution," that is, approximate values of $Z$ for small values of $\phi$, and the two solutions shall be joined. We choose as the point of junction the value of $\psi=\psi_{j}$ for which

$$
\frac{\partial^{2} u}{\partial y^{2}}=\frac{u}{2} \frac{\partial^{2}\left(u^{2}\right)}{\partial \psi^{2}}=0
$$

(inflexion point of the velocity profile). Since $\partial^{2} Z / \partial \psi^{2}=-\partial^{2}\left(u^{2}\right) / \partial \psi^{2}$, we obtain from equation (9.26)

$$
u \frac{\partial^{2}\left(u^{2}\right)}{\partial \psi^{2}}=\frac{U}{\nu} \frac{\partial Z}{\partial \phi} .
$$

To obtain a first approximation we will replace $\partial Z / \partial \phi$ by a function of $\phi$ and $u$; then we have to solve an ordinary differential equation for $u$, where the coefficients are functions of $\phi$. For $\psi=0$

$$
\frac{\partial Z}{\partial \phi}=\frac{\partial}{\partial \phi}\left[U^{2}-u^{2}\right]=2 U \frac{d U}{d \phi} ;
$$

for the point of junction, $\partial Z / \partial \phi=0$. Hence, we replace $\partial Z / \partial \phi$ by

$$
2 U \frac{d U}{d \phi}\left(1-\frac{u}{u_{j}}\right),
$$

where $u_{j}$ is the value of $u=\left(-Z^{2}+U^{2}\right)^{1 / 2}$ obtained from the outer solution.

This method was given by the writer and C. B. Millikan in a joint 
paper and applied in detail to the case in which $U$ is a linear function of $x$, that is, $U=U_{0}+U_{1} x$, where $U_{0}$ and $U_{1}$ are constants.

Other more or less laborious methods for obtaining approximate solutions of the boundary layer equations have been suggested by L. Howarth, Green, Falkner and Skan. The equations for nonstation-

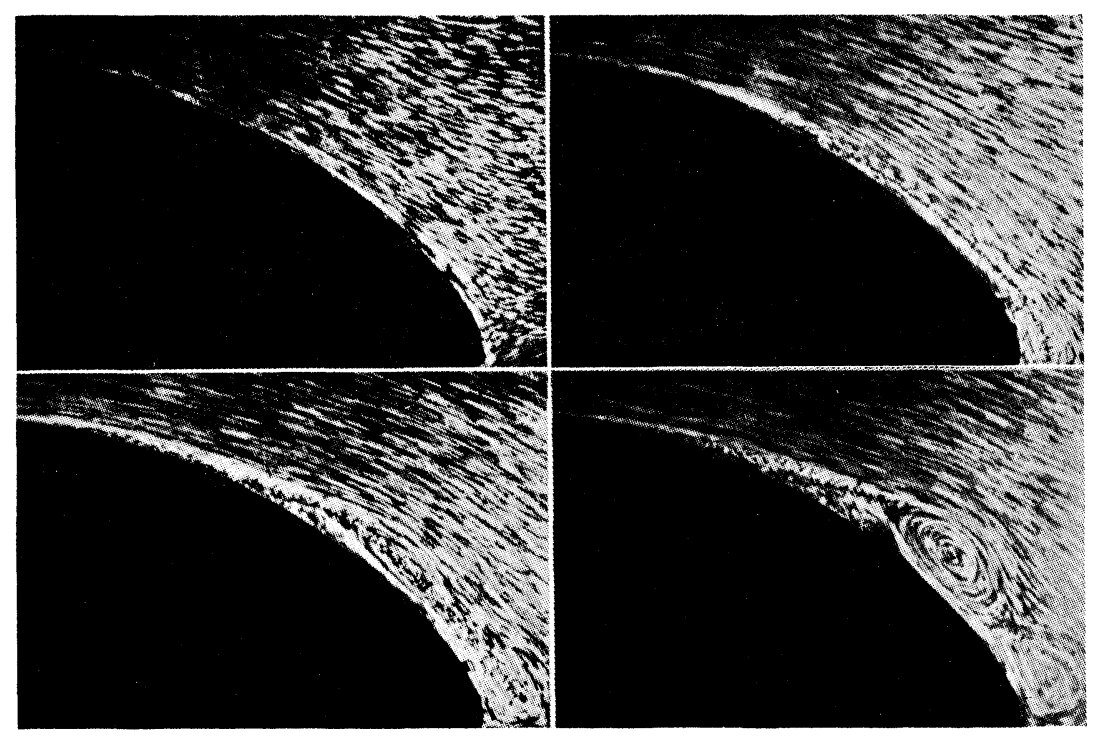

FIG. 16

From Prandtl-Tietjens, Hydro and Aeromechanics, vol. 2

ary and for three-dimensional motion have been studied by several authors and integrated in certain special cases.

One result of general importance is shown by all these investigations: The flow pattern depends largely on the sign of the function $U(d U / d x)$. If $U(d U / d x)>0$, which means that $d p / d x<0$, the thickness of the boundary layer decreases or increases slowly, and the solution of equations (9.16) can be continued without difficulty for the whole range in which $U(x)$ is given. However, if $U(d U / d x)<0$, that is, $d p / d x>0$, we soon come to a point at the surface where $\partial u / \partial y=0$. Beyond this point the streamline $\psi=0$ has two branches; the flow separates from the surface and back flow takes place between the two branches of $\psi=0$. This point is known as the separation point. It can be clearly recognized in the flow pictures shown in Fig. 16 which represent successive stages of an accelerated flow around a cy- 
lindrical body. Unfortunately, practically all the methods mentioned above converge very slowly, if at all, when we approach the separation point, and, therefore, it is very difficult to discuss the mathematical character of the solution of the boundary layer equations in the neighborhood of this point. An additional difficulty is caused by the fact that by the separation of the flow from the surface vorticity is transported into the free stream and, therefore, the assumption of irrotational motion outside the boundary is no more valid. Hence, in such cases the perturbation method fails.

Due to this failure of the method we do not get an answer for one of the fundamental questions of the hydrodynamics of real fluids, that is: What is the flow pattern of a real fluid around a submerged body in the limiting case $\nu \rightarrow 0$ ? As a matter of fact this problem is still not solved. Consider, for example, two-dimensional flow around a circular cylinder. We are not able to decide whether the flow pattern for $\nu \rightarrow 0$ approaches the potential flow of a nonviscous fluid or a stationary flow pattern consisting of a vortex-free region and a wake with continuously distributed vorticity, as suggested by Oseen, or a nonstationary flow pattern with concentrated vortex columns of alternating circulation, a flow pattern treated by the present author. It seems that we have here an example in which the analytical methods are not sufficient, at least at the present time, to solve a problem of purely analytical character. ${ }^{4}$

10. Compressible fluids. In the previous sections the fluid was assumed to be incompressible, that is, the equation of continuity or conservation of matter was used in the form

$$
\frac{\partial u_{i}}{\partial x_{i}}=0 .
$$

If we drop this restriction and denote the variable density of the fluid by $\rho$, we have

$$
\frac{\partial \rho}{\partial t}+\frac{\partial\left(\rho u_{i}\right)}{\partial x_{i}}=0
$$

${ }^{4}$ The reader may wonder why the turbulence problem has not been included in this review. This omission is due to the fact that the study of turbulent motion has not, as yet, led to a clear mathematical formulation of the fundamental laws for the mean motion. The problem of transition from laminar to turbulent motion has been discussed mathematically by several authors by means of linearized equations without reaching a satisfactory agreement with experiment. The adequate treatment of the nonlinear equations is bound to contribute essentially to the solution of this important problem. 
In this case we have five unknown quantities: the three velocity components, the density, and the pressure. To solve the problem properly we have to introduce thermodynamical concepts. We have to add the temperature as an additional unknown variable and two equations: the equation of state and the equation of the conservation of energy (mechanical plus thermal). However, in many cases the problem can be simplified by assuming a definite thermodynamic process, for example, adiabatic or isothermal change of state. This amounts to a known relation between $p$ and $\rho$. Let us assume, for example, an ideal fluid undergoing an adiabatic change of state, then we have the relation $p=$ const. $\rho^{x}$. Introducing the velocity of sound $c$ defined by $c^{2}=d p / d \rho$, we obtain the following system of equations

$$
\frac{\partial u_{i}}{\partial t}+u_{k} \frac{\partial u_{i}}{\partial x_{k}}=-\frac{c^{2}}{\rho} \frac{\partial p}{\partial x_{i}}, \quad \frac{\partial \rho}{\partial t}+\frac{\partial\left(\rho u_{i}\right)}{\partial x_{i}}=0 .
$$

These nonlinear equations can be linearized in the following cases:

(a) Assume that the velocity components $u_{i}$ and the deviation of $\rho$ from average value $\rho_{0}$ are small. In this case we obtain

$$
\frac{\partial u_{i}}{\partial t}=-\frac{c^{2}}{\rho_{0}} \frac{\partial \rho}{\partial x_{i}}, \quad \frac{\partial \rho}{\partial t}+\rho_{0} \frac{\partial u_{i}}{\partial x_{i}}=0 .
$$

The velocity of sound can be considered as constant. Eliminating the velocity components $u_{i}$, we obtain for $\rho$ the wave equation:

$$
\frac{1}{c^{2}} \frac{\partial^{2} \rho}{\partial t^{2}}=\Delta \rho
$$

(b) If the velocity components $u_{2}$ and $u_{3}$ are small whereas $u_{1}$ might be large but differs only slightly from a mean value $U$, it follows again that the deviation of the density $\rho$ from a mean value $\rho_{0}$ is small. Then putting $u_{1}=U+u_{1}^{\prime}, u_{2}=u_{2}^{\prime}, u_{3}=u_{3}^{\prime}$ we obtain

$$
\frac{\partial u_{i}^{\prime}}{\partial t}+U \frac{\partial u_{i}^{\prime}}{\partial x_{1}}=-\frac{c^{2}}{\rho_{0}} \frac{\partial \rho}{\partial x_{i}}, \quad \frac{\partial \rho}{\partial t}+U \frac{\partial \rho}{\partial x_{1}}+\rho_{0} \frac{\partial u_{i}^{\prime}}{\partial x_{i}}=0 .
$$

The velocity of sound can again be considered constant, so that eliminating the velocity components $u_{i}^{\prime}$ we have

$$
\frac{1}{c^{2}}\left(\frac{\partial}{\partial t}+U \frac{\partial}{\partial x_{1}}\right)^{2} \rho=\Delta \rho
$$

Let us consider the stationary case, that is, $\partial \rho / \partial t=0$. Then we have 


$$
\left(1-\frac{U^{2}}{c^{2}}\right) \frac{\partial^{2} \rho}{\partial x_{1}^{2}}+\frac{\partial^{2} \rho}{\partial x_{2}^{2}}+\frac{\partial^{2} \rho}{\partial x_{3}^{2}}=0 .
$$

It is evident that the linear differential equation (10.8) is of the elliptic type if $U<c$ (subsonic flow) and of the hyperbolic type, if $U>c$ (supersonic flow). In the first case equation (10.8) is reduced to Laplace's equation by the affine transformation

$$
x_{1}^{\prime}=\frac{x_{1}}{\left(1-U^{2} / c^{2}\right)^{1 / 2}}, \quad x_{2}^{\prime}=x_{2}, \quad x_{3}^{\prime}=x_{3} .
$$

If $U>c$, equation (10.8) is of the type of the wave equation and can be integrated by use of well known methods developed for the solution of this equation.

Equations (10.5) and (10.8) are often used for approximate solution of the system of equations (10.3). However, in many cases these approximate solutions do not describe the physical picture closely enough and we are forced to search for methods of solving the nonlinear equations.

Let us consider stationary irrotational two-dimensional flow, using the symbols $x$ and $y$ for the coordinates, and $u$ and $v$ for the velocity components. Then denoting the potential function by $\phi(x, y)$ we obtain, by putting $\partial \rho / \partial t=\partial u / \partial t=\partial v / \partial t=0$ and eliminating $\rho$ from equations (10.3),

$$
\left(1-\frac{u^{2}}{c^{2}}\right) \frac{\partial^{2} \phi}{\partial x^{2}}-2 \frac{u v}{c^{2}} \frac{\partial^{2} \phi}{\partial x \partial y}+\left(1-\frac{v^{2}}{c^{2}}\right) \frac{\partial^{2} \phi}{\partial y^{2}}=0 .
$$

In this equation $c^{2}$ is a given function of $q^{2}=u^{2}+v^{2}$. Namely, according to the definition of the velocity of sound $c^{2}$ is a given function of $\rho$; on the other hand, it follows from (10.3) that

$$
d\left(u^{2}+v^{2}\right)+\frac{c^{2}}{\rho} d \rho=0 .
$$

Let us apply Legendre's transformation introducing the velocity components $u$ and $v$ as independent variables and $X=x u+y v-\phi$ as the unknown function. We obtain

$$
\left(1-\frac{u^{2}}{c^{2}}\right) \frac{\partial^{2} X}{\partial v^{2}}+2 \frac{u v}{c^{2}} \frac{\partial^{2} X}{\partial u \partial v}+\left(1-\frac{v^{2}}{c^{2}}\right) \frac{\partial^{2} X}{\partial u^{2}}=0 .
$$

If in the whole domain occupied by the fluid $u^{2}+v^{2}>c^{2}$, equation (10.12) is of the hyperbolic type and has fixed real characteristics in the $u, v$ plane. Therefore, in this case the integration of equation 
(10.12) can be achieved by the methods of characteristics. For the construction of the characteristics it is convenient to use the polar coordinates $q=\left(u^{2}+v^{2}\right)^{1 / 2}$ and $\theta=\tan ^{-1} v / u$ in the $u, v$ plane. In these coordinates the differential equation of the characteristic curves becomes

$$
\frac{1}{q} \frac{d q}{d \theta}=\frac{c}{\left(q^{2}-c^{2}\right)^{1 / 2}}
$$

Since $c$ is a function of $q$ only, equation (10.13) is a differential equation of the first order for $q(\theta)$. Its solution consists of two families

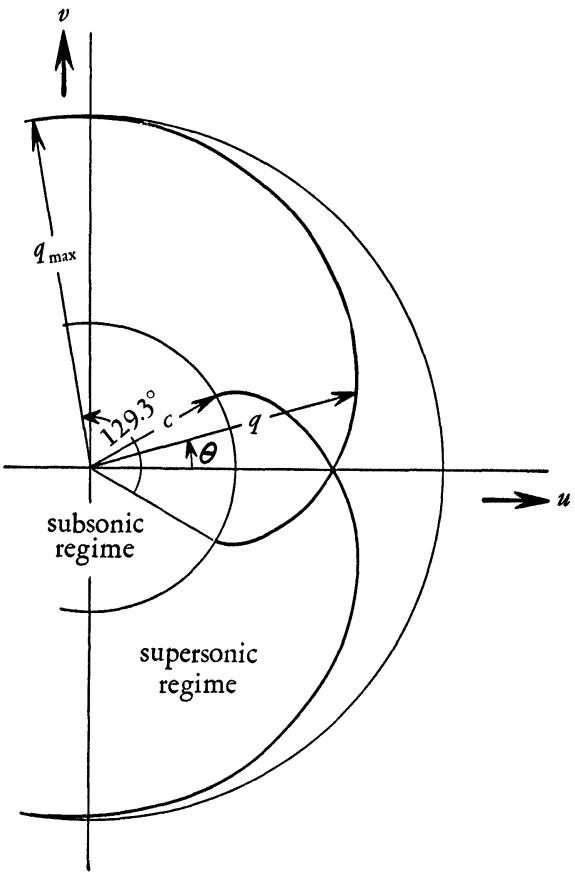

FIG. 17

of curves: one family corresponds to the positive, the other to the negative sign of the square root. If the isothermal process is assumed, $c=$ const. and the characteristics are two families of involutes of the circle of the radius $c$ covering the whole domain of the $u, v$ plane outside of this circle. If, according to the so-called polytropic law, $p$ is proportional to $\rho^{k}$, we obtain epicycloids which touch the two circles whose radii are equal to the minimum value of $c$ and the maximum value of $q$ respectively. The characteristic curves are indicated in Fig. 17. If the characteristics of (10.12) in the $u, v$ plane are known, 
the next step consists of constructing the corresponding characteristic curves of (10.10) in the $x, y$ plane. L. Prandtl and A. Busemann have worked out graphical methods for this purpose.

It is a characteristic property of equation (10.10) that in the supersonic range it can have discontinuous solutions. Both density and velocity change discontinuously at certain surfaces. Such discontinuities occurring in supersonic flow are known as shock waves; they also can be considered as waves of finite amplitude if we look at the phenomenon from the standpoint of an observer moving with the fluid. B. Riemann was the first who pointed out (in 1860) that in a compressible fluid waves of finite amplitude can produce discontinuities. Later W. J. M. Rankine, H. Hugoniot, J. Hadamard, E. Jouguet, G. I. Taylor and others, worked out in detail the theory of the discontinuous solutions. Engineering applications of the theory of shock waves were made by A. Stodola, L. Prandtl, and many other authors. Applications to the ballistic problem were made by E. Mach, C. Cranz, G. I. Taylor, J. W. Maccoll, and P. S. Epstein.

If in the whole domain $u^{2}+v^{2}=q^{2}<c^{2}$, the problem of integration of equation (10.10) can be attacked by the method of successive approximations. Let us consider the classical example of the two-dimensional flow of a compressible fluid around a circular cylinder. We assume that the velocity of the flow at large distance from the obstacle is parallel to the $x$ axis and has the magnitude $U$. We denote the velocity of sound corresponding to the density and pressure prevailing in the undisturbed flow by $c_{0}$ and the exponent of the polytropic law by $\kappa$. Then the velocity of sound at an arbitrary point, where the magnitude of the velocity is equal to $q$, is given by

$$
c^{2}=c_{0}^{2}+\frac{\kappa-1}{2}\left(U^{2}-q^{2}\right)
$$

Introducing this relation into equation (10.10) and using polar coordinates $r$ and $\theta$, we obtain

$$
\begin{aligned}
& {\left[1-\frac{1}{2}(\kappa-1) M^{2}\left(\frac{q^{2}}{U^{2}}-1\right)\right] \Delta \phi} \\
& =\frac{1}{2} \frac{M^{2}}{U^{2}}\left(\frac{\partial \phi}{\partial r} \frac{\partial q^{2}}{\partial r}+\frac{1}{r^{2}} \frac{\partial \phi}{\partial \theta} \frac{\partial q^{2}}{\partial \theta}\right)
\end{aligned}
$$

where $M=U / c_{0}$ is called Mach's number and

$$
q^{2}=\left(\frac{\partial \phi}{\partial r}\right)^{2}+\frac{1}{r^{2}}\left(\frac{\partial \phi}{\partial \theta}\right)^{2}
$$


We assume that $\phi$ can be developed in a series of ascending powers of $M^{2}$ :

$$
\phi=\phi_{0}+M^{2} \phi_{1}+M^{4} \phi_{2}+\cdots .
$$

Obviously, we obtain from equation (10.15)

$$
\Delta \phi_{0}=0 .
$$

It is seen that $\phi_{0}(r, \theta)$ is the solution for incompressible fluid. We put the radius of the circle equal to unity. Then the boundary condition is $\partial \phi_{0} / \partial r=0$ for $r=1$, and we obtain:

$$
\phi_{0}=(r+1 / r) \cos \theta .
$$

We also develop $q^{2}$ in ascending powers of $M^{2}$ by writing

$$
q^{2}=q_{0}^{2}+M^{2} q_{1}^{2}+M^{2} q_{2}^{4}+\cdots .
$$

Substituting the expressions (10.16) and (10.19) in equation (10.15) and equating the terms multiplied by the same power of $M$, we have the following relations for $\phi_{1}, \phi_{2}, \ldots$

$$
\begin{aligned}
\Delta \phi_{1}= & \frac{1}{2 U^{2}}\left(\frac{\partial \phi_{0}}{\partial r} \frac{\partial q_{0}^{2}}{\partial r}+\frac{1}{r^{2}} \frac{\partial \phi_{0}}{\partial \theta} \frac{\partial q_{0}^{2}}{\partial \theta}\right), \\
\Delta \phi_{2}= & \frac{1}{2 U^{2}}(k-1)\left(q_{0}^{2}-1\right) \Delta \phi_{1} \\
& +\frac{1}{2 U^{2}}\left(\frac{\partial \phi_{0}}{\partial r} \frac{\partial q_{1}^{2}}{\partial r}+\frac{1}{r^{2}} \frac{\partial \phi_{0}}{\partial \theta} \frac{\partial q_{1}^{2}}{\partial \theta}\right) \\
& +\frac{1}{2 U^{2}}\left(\frac{\partial \phi_{1}}{\partial r} \frac{\partial q_{0}^{2}}{\partial r}+\frac{1}{r^{2}} \frac{\partial \phi_{1}}{\partial \theta} \frac{\partial q_{0}^{2}}{\partial \theta}\right) .
\end{aligned}
$$

The boundary conditions are $\partial \phi / \partial r=0$ for $r=1$ and $\partial \phi_{1} / \partial r=\partial \phi_{2} / \partial r$ $=0$ for $r=\infty$.

The method sketched here is essentially identical to that used by Lord Rayleigh, O. Janzen, L. Poggi, C. Kaplan, I. Ismai, K. Tamada and Y. Saito. The calculations of the Japanese authors are the most reliable ones; they corrected some numerical errors which occurred in previous publications. G. I. Taylor worked out an experimental method for solving the same problem by successive approximations. He employed an electric device analogous to that used for the experimental solution of Laplace's equation.

Another method for solving problems of subsonic flow in compress- 
ible fluids was suggested by P. Molenbroek and A. Chaplygin. These authors use a transformation of independent variables which is analogous to the Legendre transformation mentioned before in this section. However, instead of using the potential $\phi(x, y)$ and its Legendre transform $X(u, v)$ as unknown functions they use $\phi(x, y)$ and the stream function $\psi(x, y)$ which satisfies identically the continuity equation for stationary flow:

$$
\frac{\partial(\rho u)}{\partial x}+\frac{\partial(\rho v)}{\partial y}=0 .
$$

We write $\rho u=\partial \psi / \partial y$ and $\rho v=-\partial \psi / \partial x$. Then we have

$$
d \phi=u d x+v d y, \quad d \psi=-\rho v d x+\rho u d y .
$$

We resolve these equations for $d x$ and $d y$ and introduce as independent variables polar coordinates in the $u$, $v$ plane, namely, $q=\left(u^{2}+v^{2}\right)^{1 / 2}$ and $\theta=\tan ^{-1} v / u$. Substituting

$d \phi=\frac{\partial \phi}{\partial q} d q+\frac{\partial \psi}{\partial \theta} d \theta, \quad d \psi=\frac{\partial \psi}{\partial q} d q+\frac{\partial \psi}{\partial \theta} d \theta, \quad u=q \cos \theta, \quad v=q \sin \theta$ into equations (10.22) and resolving them for $d x$ and $d y$, we obtain

$$
\begin{aligned}
& d x=\left(\frac{\cos \theta}{q} \frac{\partial \phi}{\partial q}+\frac{\sin \theta}{q \rho} \frac{\partial \psi}{\partial q}\right) d q+\left(\frac{\cos \theta}{q} \frac{\partial \phi}{\partial \theta}+\frac{\sin \theta}{q \rho} \frac{\partial \psi}{\partial \theta}\right) d \theta, \\
& d y=\left(-\frac{\sin \theta}{q} \frac{\partial \phi}{\partial q}+\frac{\cos \theta}{q \rho} \frac{\partial \psi}{\partial q}\right) d q-\left(\frac{\sin \theta}{q} \frac{\partial \phi}{\partial \theta}+\frac{\cos \theta}{q \rho} \frac{\partial \psi}{\partial \theta}\right) d \theta .
\end{aligned}
$$

The relations

$$
\frac{\partial}{\partial \theta}\left(\frac{\partial x}{\partial q}\right)=\frac{\partial}{\partial q}\left(\frac{\partial x}{\partial \theta}\right), \quad \frac{\partial}{\partial \theta}\left(\frac{\partial y}{\partial q}\right)=\frac{\partial}{\partial q}\left(\frac{\partial y}{\partial \theta}\right)
$$

furnish the following two relations between the derivatives $\partial \phi / \partial q$, $\partial \phi / \partial \theta$ and $\partial \psi / \partial q, \partial \psi / \partial \theta$ :

$$
\begin{aligned}
& -\sin \theta \frac{\partial \phi}{\partial q}+\frac{1}{\rho} \cos \theta \frac{\partial \psi}{\partial q}=-\frac{\cos \theta}{q} \frac{\partial \phi}{\partial \theta}-\frac{\sin \theta}{q \rho}\left(1+\frac{q}{\rho} \frac{d \rho}{d q}\right) \frac{\partial \psi}{\partial \theta} \\
& -\cos \theta \frac{\partial \phi}{\partial q}-\frac{1}{\rho} \sin \theta \frac{\partial \psi}{\partial q}=\frac{\sin \theta}{q} \frac{\partial \phi}{\partial \theta}-\frac{\cos \theta}{q \rho}\left(1+\frac{q}{\rho} \frac{d \rho}{d q}\right) \frac{\partial \psi}{\partial \theta}
\end{aligned}
$$

Multiplying the first equation of (10.24) by $\cos \theta$ and the second by $\sin \theta$, we have by subtraction 


$$
\frac{1}{\rho} \frac{\partial \psi}{\partial q}=-\frac{1}{q} \frac{\partial \phi}{\partial \theta} .
$$

Multiplying the first equation by $\sin \theta$ and the second by $\cos \theta$, we obtain by addition

$$
\frac{\partial \phi}{\partial q}=\frac{1}{q \rho}\left(1+\frac{q}{\rho} \frac{d \rho}{d q}\right) \frac{\partial \psi}{\partial \theta} .
$$

From equation (10.11) follows $(q / \rho) d \rho / d q=-q^{2} / c^{2}$; hence, equation (10.26) becomes

$$
\frac{\partial \phi}{\partial q}=\frac{1}{q \rho}\left(1-\frac{q^{2}}{c^{2}}\right) \frac{\partial \psi}{\partial \theta} .
$$

Eliminating, for example, $\phi$ from equations (10.25) and (10.27), we obtain the following linear differential equation for $\psi$ :

$$
\frac{q \rho}{1-q^{2} / c^{2}} \frac{\partial}{\partial q}\left(\frac{q}{\rho} \frac{\hat{c} \psi}{\partial q}\right)+\frac{\partial^{2} \psi}{\partial \theta^{2}}=0,
$$

where $c$ is given as function of $q$ by means of the relations $c^{2}=d p / d \rho$, $d p / \rho+q d q=0$ and $p=f(\rho)$. The last relation depends on the law of the change of state of the fluid.

Chaplygin has shown that the differential equation (10.29) can be reduced to Laplace's equation by an appropriate choice of the relation $p=f(\rho)$. Laplace's equation for the polar coordinates $q$ and $\theta$ has the form

$$
q \frac{\partial \psi}{\partial q}\left(q \frac{\hat{c} \psi}{\partial q}\right)+\frac{\partial^{2} \psi}{\partial \theta^{2}}=0 .
$$

Equation (10.29) is reduced to the form (10.29) if

$$
\frac{d q}{q}=\frac{1}{a} \frac{\rho d q}{q}=a \frac{\left(1-q^{2} / c^{2}\right)}{q \rho} d q
$$

where $a$ is an arbitrary constant. Hence, we have to choose $p=f(\rho)$ such that

$$
\rho^{2}=a^{2}\left(1-q^{2} / c^{2}\right)
$$

or

$$
q^{2}=c^{2}-\frac{\rho^{2} c^{2}}{a^{2}} .
$$


Since $d\left(q^{2}\right) / d \rho=-2 c^{2} / \rho$, we obtain by differentiation of (10.31) the relation

$$
\frac{d\left(c^{2}\right)}{d \rho}=-2 \frac{c^{2}}{\rho} .
$$

From equation (10.32) follows $c^{2}=d p / d \rho=B / \rho^{2}$ and

$$
p=A-\frac{B}{\rho} \text {. }
$$

Introducing the specific volume $V=1 / \rho$, we can write equation (10.33) in the form

$$
p-p_{0}=\text { const. }\left(V_{0}-V\right) \text {. }
$$

It is seen that we obtain Laplace's equation for $\psi$ (and also for $\phi$ ) if the polytropic curve in the $p, V$ plane is replaced by a straight line.

It is obvious that if the density change is small the adiabatic or other polytropic curves can be approximated by straight lines. A. Chaplygin, D. Riabouchinsky, B. Demtschenko, J. Caius and A. Busemann applied this method of approximation to the slow motion of a compressible fluid. It seems to the writer that an application to the flow of a compressible fluid with a velocity which is comparable to the velocity of sound is of greater practical interest. Let us assume that the undisturbed velocity of the fluid is $U$ (where $U<c$ ) and the density corresponding to the velocity is $\rho_{0}=1 / V_{0}$. If we assume that the change of the density is sufficiently small so that the pressure can be expanded as function of $V-V_{0}$ and approximated by a linear term, it is possible to determine possible flow patterns, velocity and pressure distributions in compressible fluids by use of the methods of conformal transformation. By means of successive approximations, which require considerably less labor than Rayleigh's method mentioned before in this section, it is also possible to determine the velocity and pressure distribution around given body. H. S. Tsien worked out this application of Chaplygin's method upon suggestion of the present writer.

The problem of flow of a compressible fluid around a cylindrical body or a body of axial symmetry is of great practical importance in view of the increasing speed of aircraft, which already have flown faster than 2/3 of the velocity of sound. Experimental evidence seems to show that if the velocity at any point of the wing surface reaches the velocity of sound, a shock wave appears. However, G. I. Taylor calculated numerically with great patience an analogous flow prob- 
lem, namely, the flow of a compressible fluid through a nozzle, whose cross section decreases to a minimum value and again increases. He obtained a flow pattern, showing near the walls a velocity greater than that of sound, but no discontinuity. Another important case is

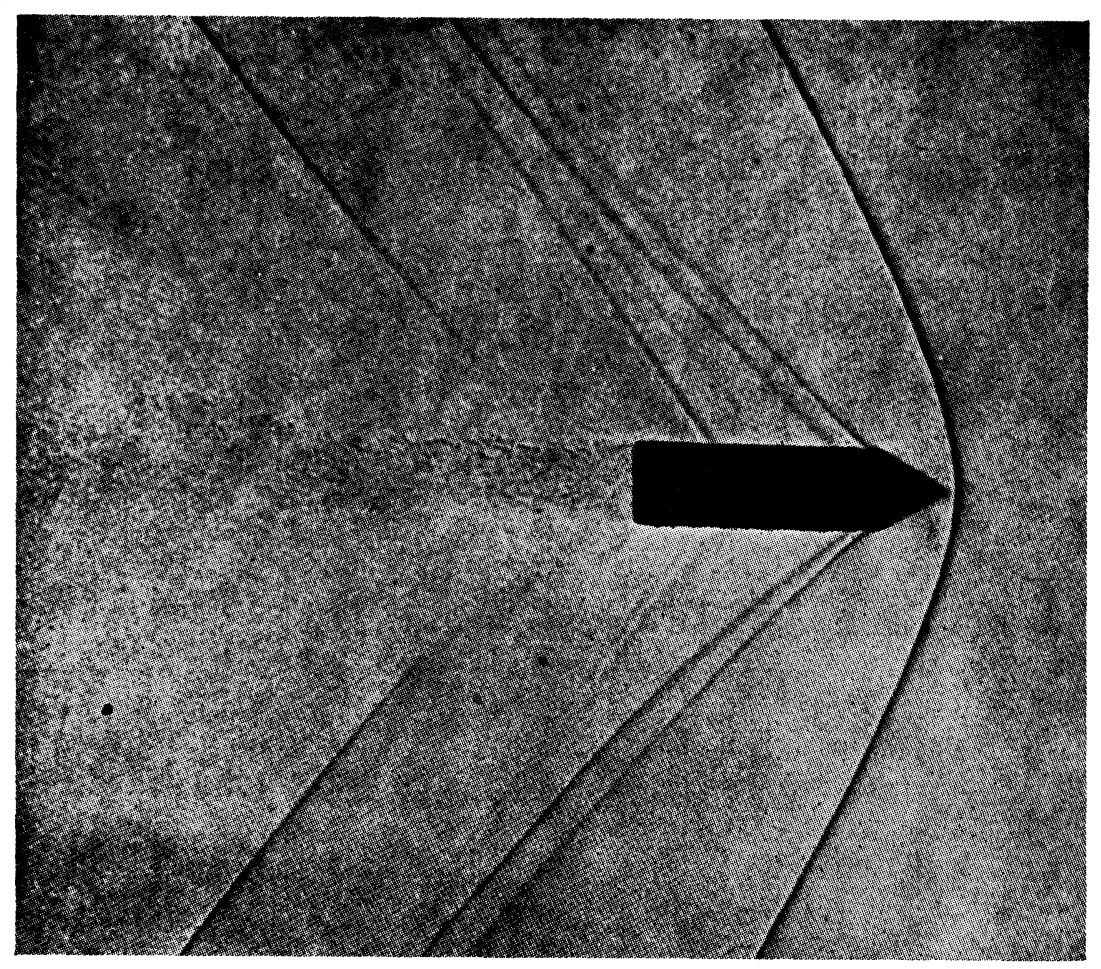

FIG. 18a. Photograph of a bullet in flight. From G. I. Taylor and J. W. Maccoll, Aerodynamic Theory, vol. 3

the motion of a blunt-shaped body at supersonic velocity. Observations show that a curved discontinuity surface is created in front of the body; between this surface and the blunt nose of the body the flow is subsonic and the velocity becomes zero at the nose of the body. The same phenomenon occurs also in the case of a body with a pointed nose or leading edge at velocities slightly higher than the velocity of sound (Fig. 18a), whereas if the speed is sufficiently large, the shock wave starts from the pointed edge, or in the case of a body of rotation from the apex (Fig. 18b). This problem is important in ballistics; for example, the so-called terminal velocity of aerial bombs 
dropped from large heights is in this range. Unfortunately, no method as yet is available for solving such mixed subsonic-supersonic prob-

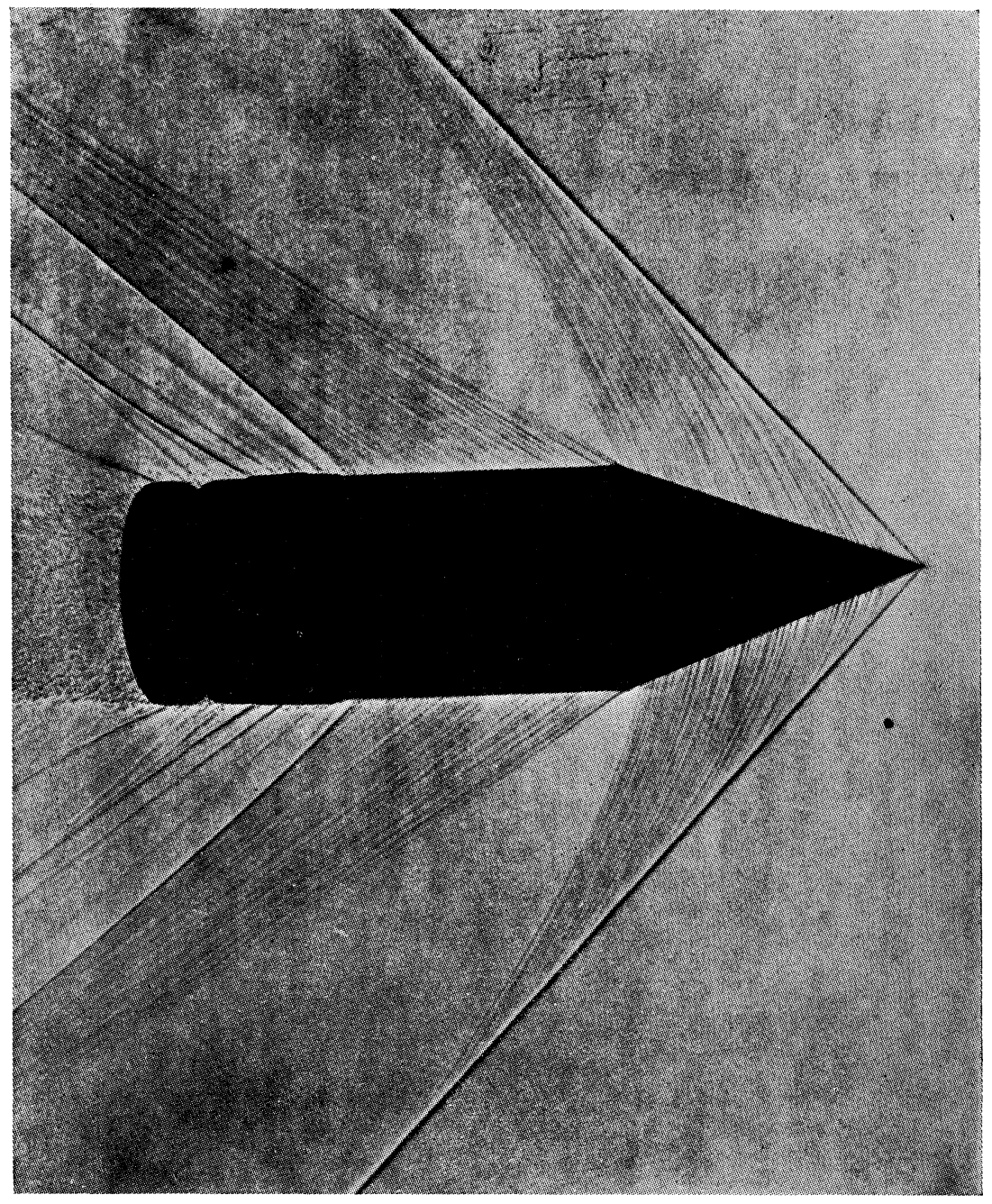

FIG. 18b. Photograph of a conical headed projectile in flight.

Photograph by T. Harris, British Ordnance Committee

lems. The difficulty is that the differential equation for the potential or the stream function is of the elliptic type in one part of the domain and of the hyperbolic type in the other. It seems to the writer that 
the invention of methods for the solution of such mixed problems would be an achievement both from a practical and a mathematical point of view.

An attempt was made in this paper to show the applications of analytical methods available for the solution of certain nonlinear problems in which the engineer is interested. Some gaps were shown and frontiers indicated beyond which the safe guidance of the mathematical analysis is for the time being lacking. I would consider this lecture a great success if it would induce mathematicians to volunteer for pioneering work in pushing those frontiers "farther west."

\section{BiBLIOGRAPHY}

\section{SELF-EXCITED NONLINEAR OSCILlations}

1. B. Van der Pol, Relaxation oscillations, Philosophical Magazine, vol. 2 (1926), p. 978. Frequency demultiplication, Nature, September 10, 1927.

2. M. A. Andronow, Les cycles limites de Poincare et la theorie des oscillations auto-entretenues, Comptes Rendus de l'Académie des Sciences, Paris, vol. 189 (1929), pp. 559-561.

3. M. A. Andronow and A. Witt, Sur la theorie mathematique des auto-oscillations, ibid., vol. 190 (1930), pp. 256-258.

4. A. M. Lienard, Oscillations auto-entretenues, Proceedings of the Third International Congress of Applied Mechanics (Stockholm, 1930), vol. 3 (1930), pp. 173-177.

5. B. Van der Pol, The nonlinear theory of electric oscillations, Proceedings of the Institute of Radio Engineers, vol. 22 (1934), pp. 1051-1086.

6. J. Haag, Sur les oscillations auto-entretenues, Comptes Rendus de l'Académie des Sciences, Paris, vol. 199 (1934), pp. 906-909. Sur l'étude asymptotique des oscillations de relaxation, ibid., vol. 202 (1936), pp. 102-104. Sur la theorie des oscillations de relaxation, ibid., vol. 204 (1937), pp. 932-934. Formules asymptotiques concernant les oscillations de relaxation, ibid., vol. 206 (1938), pp. 1235-1237.

\section{SUBHARMONIC RESONANCE}

1. F. Melde, Über die Erregung stehender Wellen eines fadenförmigen Körpers, Foggendorffs Annalen der Physik und Chemie, (4), vol. 19 (1880), pp. 193-215.

2. Lord Rayleigh, On the maintenance of vibrations by forces of double frequency and on the propagation of waves through a medium endowed with periodic structure, Philosophical Magazine, (5), vol. 24 (1887), pp. 145-159. Theory of Sound, \#68b, 2d edition, 1894, pp. 81-85.

3. A. Stephenson, On a class of forced oscillations, Quarterly Journal of Mathematics, vol. 37 (1906), pp. 353-360.

4. C. V. Raman, The small motion at the modes of a vibrating string, Physical Review, vol. 32 (1911), pp. 309-315.

5. - - On the Maintenance of Combinational Vibrations by Two Simple Harmonic Forces, Bulletin no. 11, Indian Association for the Cultivation of Science, 1914, pp. 1-24.

6. - On Motion in a Periodic Field of Force, Bulletin no. 11, Indian Association for the Cultivation of Science, 1194, pp. 25-42. 
7. A. Dey and C. V. Raman, On the maintenance of vibrations by a periodic field of force, Proceedings of the Indian Association for the Cultivation of Science, vol. 2 (1917), pp. 1-15.

8. G. Duffing, Erwungene Schwingungen bei veränderlicher Eigenfrequenz und ihre technische Bedeutung, Braunschweig, 1918.

9. D. Banerji, On the theory and some applications of sub-synchronous pendulums, Proceedings of the Indian Association for the Cultivation of Science, vol. 7 (1921-1922) pp. 145-158.

10. L. Amaduzzi, Le nodali di Savart ed il suono rauco, Rendiconti del Accademia delle Scienze dell' Istituto di Bologna, new series, vol. 29 (1924-1925), pp. 73-76.

11. L. Mandelstam and N. Papalexi, Über Resonanzerscheinungen bei Frequenzteilung, Zeitschrift für Physik, vol. 73 (1931), pp. 233-248.

12. N. Kryloff and N. Bogoliuboff, Über einige Methoden der nicht-linearen Mechanik in ihren Anwendungen auf der nicht-linearen Resonanz, Schweizerische Bauzeitung, vol. 103 (1934), pp. 255-257, 267-270. (This paper contains a list of 14 papers of these authors on the subject of nonlinear vibrations.)

13. P. O. Pederson, Sub-harmonics in forced oscillations in dissipative systems, Journal of the Acoustical Society of America, vol. 6 (1935), pp. 227-238; vol. 7 (1935), pp. $64-70$

\section{LARGE DEFLECTION THEORY}

1. Th. von Kármán, Article in Encyklopädie der mathematischen Wissenschaften, vol. $\mathrm{IV}_{4}(1910)$, p. 349

2. — Untersuchungen über Knickfestigkeit, Forschungsarbeiten, no. 81, 1910.

3. R. Grammel, Das Umstiulpen und Umkippen von elastischen Ringen, Zeitschrift für angewandte Mathematik und Mechanik, vol. 3 (1923), pp. 429-441.

4. Th. von Kármán, E. E. Sechler and L. H. Donnell, The strength of thin plates in compression, Transactions of the American Society of Mechanical Engineers, vol. 54 (1932), pp. 53-57.

5. H. L. Cox, The Buckling of Thin Plates in Compression, British Aeronautical Research Committee, Reports and Memoranda, no. 1554, 1933.

6. S. Way, Bending of circular plates with large deflection, Transactions of the American Society of Mechanical Engineers, vol. 56 (1934), pp. 627-633.

7. C. B. Biezeno, Über die Bestimmung der Durchschlagkraft einer schwach gekrümmten kreisförmigen Platte, Zeitschrift für angewandte Mathematik und Mechanik, vol. 15 (1935), pp. 13-30.

8. K. Marguerre and E. Trefftz, Über die Tragfähigkeit eines Plattenstreifens nach Überschreiten der Beullast, Zeitschrift für angewandte Mathematik und Mechanik, vol. 17 (1937), pp. 85-100.

9. M. Yamamoto and K. Kondo, Buckling and Failure of Thin Rectangular Plates in Compression, Reports of the Aeronautical Research Institute of the Tokyo University, no. 119, 1937.

10. C. B. Biezeno, Über eine Stabilitätsfrage beim gelenkig gelagerten schwach gekrümmten Stab, Zeitschrift für angewandte Mathematik und Mechanik, vol. 18 (1938), pp. 21-30.

11. R. Grammel, Das Durchschlagen von Kreisringen, Ingenieur-Archiv, vol. 9 (1938), pp. 126-137.

12. A. Kromm and K. Marguerre, Verhalten eines von Schub und Druckkräften beanspruchten Plattenstreifens oberhalb der Beulgrenze, Jahrbuch Deutschen Versuchanstalt für Luftfahrt, 1938, pp. 263-275.

13. K. Marguerre, Über die Behandlung von Stabilitätsproblemen mit Hilfe der 
energetischen Methode, Zeitschrift für angewandte Mathematik und Mechanik, vol. 18 (1938), pp. 57-73.

14. - Die Durchschlagskraft eines schwach gekrümmten Balkens, Sitzungsberichte der Berliner mathematischen Gesellschaft, vol. 37 (1938), pp. 22-40.

15. S. Way, "A Laterally Loaded Clamped Square Plate with Large Deflection," Contributions to the Mechanics of Solids, S. Timoshenko 60th Anniversary Volume, McGraw-Hill, 1938, pp. 240-256.

16. K. O. Friedrichs and J. J. Stoker, The non-linear boundary value problem of the buckled plate, Proceedings of the National Academy of Sciences, vol. 25 (1939), pp. $535-540$.

17. Th. von Kármán and Hsue-shen Tsien, The buckling of spherical shells by external pressure, Journal of the Aeronautical Sciences, vol. 7 (1939), pp. 43-50.

\section{THEORY OF ELASTICITY WITH FINITE DEFORMATIONS}

1. G. Kirchhoff, Über die Gleichungen des Gleichgewichtes eines elastischen Körpers bei nicht unendlich kleinen Verschiebungen seiner Theile, Sitzungsberichte der mathematischnaturwissenschaftlichen Klasse der Akademie der Wissenschaften, Vienna, vol. 9 (1852), pp. 762-773.

2. J. Boussinesq, Theorie des ondes liquides periodiques, Mémoires Presentées . . à l'Académie des Sciences de Bruxelles, Sciences Mathématiques et Physiques, vol. 20 (1872), pp. 509-615.

3. M. Brillouin, Deformations homogènes finies; énergie d'un corps isotrope, Comptes Rendus de l'Académie des Sciences, Paris, vol. 112 (1891), pp. 1500-1502.

4. E. Cosserat and F. Cosserat, Sur la theorie de l'elasticite, Annales de la Faculté des Sciences de l'Université de Toulouse, vol. 10 (1896).

5. E. Trefftz, Zur Theorie der Stabilität des elastischen Gleichgewichts, Zeitschrift für angewandte Mathematik und Mechanik, vol. 13 (1933), pp. 160-165.

6. F. D. Murnaghan, Finite deformations of an elastic solid, American Journal of Mathematics, vol. 59 (1937), pp. 235-260.

7. M. Biot, Theory of elasticity with large displacements and rotations, Proceedings of the Fifth International Congress of Applied Mechanics, 1938, pp. 117-122. Nonlinear theory of elasticity and the linearized case for a body under initial stress, Philosophical Magazine, (7), vol. 27 (1939), pp. 468-489.

8. R. Kappus, Zur Elastizitätstheorie endlicher Verschiebungen. Zeitschrift für angewandte Mathematik und Mechanik, vol. 19 (1939), pp. 271-285, 344-361.

\section{STRESS DISTRIBUTION IN PLASTIC AND NONCOHERENT MASS}

1. W. J. M. Rankine, On the stability of loose earth, Philosophical Transactions of the Royal Society, 1857, pp. 9-27.

2. J. Boussinesq, Sur les modes d'équilibre limite les plus simples, que peut présenter un massif sans cohésion fortement comprimé, Comptes Rendus de l'Académie des Sciences, Paris, vol. 80 (1874), p. 546.

3. F. Kötter, Die Entwicklung der Lehre vom Erddruck, Jahresbericht der Deutschen Mathematiker-Vereinigung, vol. 2 (1893), p. 77.

4. - Die Bestimmung des Druckes an gekrümmten Flächen, eine Aufgabe aus der Lehre vom Erddruck, Bericht der Berliner Akademie der Wissenschaften, 1903, p. 229.

5. ——, Über den Druck von Sand ..., Bericht der Berliner Akademie der Wissenschaften, 1909, p. 493.

6. A. Haar and Th. von Kármán, Zur Theorie der Spannungszustände in plastischen 
und sandartigen Medien, Nachrichten der Gesellschaft der Wissenschaften zu Göttingen, mathematisch-physikalischer Klasse, 1909, pp. 204-218.

7. H. Reissner, Theorie des Erddruckes, Enzyklopädie der mathematischen Wissenschaften, vol. $\mathrm{IV}_{4}(1909)$, p. 387.

8. L. Prandtl, Über die Eindringungsfestigkeit (Härte) plastischer Baustoffe und die Festigkeit von Schneiden, Zeitschrift für angewandte Mathematik und Mechanik, vol. 1 (1921), pp. 15-20.

9. H. Hencky, Über einige statisch bestimmte Fälle des Gleichgewichts in plastischen Körpern, ibid., vol. 3 (1923), pp. 241-251.

10. L. Prandtl, Anwendungsbeispiele zu einem Henckyschen Satz über das plastische Gleichgewicht, ibid., vol. 3 (1923), pp. 401-406.

11. C. Carathéodory and E. Schmidt, Über die Hencky-Prandtlschen Kurven, ibid., vol. 3 (1923), pp. 468-475.

12. H. Hencky, Zur Theorie plastischer Deformationen und der hierdurch im Material hervorgerufenen Nachspanungen, ibid., vol. 4 (1924), pp. 323-334.

13. H. Reissner, Zum Erddruckproblem, Proceedings of the First International Congress of Applied Mechanics (Delft, 1924), pp. 295-311.

14. H. Hencky, Über langsame stationäre Strömungen in plastischen Massen mit Rücksicht auf die Vorgänge beim Walzen, Pressen und Ziehen von Metallen, Zeitschrift für angewandte Mathematik und Mechanik, vol. 5 (1925), pp. 115-124.

15. —- Die Bewegungsgleichungen beim nichtstationären Fliessen plastischer Massen, ibid., vol. 5 (1925), pp. 144-146.

16. R. von Mises, Bemerkungen zur Formulierung des mathematischen Problems der Plastizitätstheorie, ibid., vol. 5 (1925), pp. 147-149.

17. E. Trefftz, Über die Spannungsverteilung in tordierten Stäben bei teilweiser Überschreitung der Fliessgrenze, ibid., vol. 5 (1925), pp. 64-73.

18. Th. von Kármán, Über elastische Grenzzustände, Proceedings of the Second International Congress of Applied Mechanics (Zürich, 1926), pp. 23-32.

19. W. Jenne, Räumliche Spannungsverteilungen in festen Körpern bei plastischer Deformation, Zeitschrift für angewandte Mathematik und Mechanik, vol. 8 (1928), pp. 18-44.

20. F. Löbell, Zum Problem der Hauptschubspannungslinien in plastischen Stoffen, ibid., vol. 9 (1929), pp. 213-225.

21. A. Reuss, Berïcksichtigung der elastischen Formänderung in der Plastizitätstheorie, ibid., vol. 10 (1930), pp. 266-274.

22. W. Prager, Der Einfluss der Verformung auf die Fliessbedingung zähplastischer Körper, ibid., vol. 15 (1935), pp. 76-80.

23. E. Melan, Zur Plastizität des räumlichen Kontinuums, Ingenieur-Archiv, vol. 9 (1938), pp. 116-126.

\section{FLUID JETS}

1. H. Helmholtz, Über diskontinuierliche Flïssigkeitsbewegungen, Monatsberichte der Berliner Akademie, 1868.

2. G. Kirchhoff, Zur Theorie freier Flïssigkeitsstrahlen, Journal für die reine und angewandte Mathematik, vol. 70 (1869), p. 289.

3. Lord Rayleigh, On the resistance of fluids, Philosophical Magazine, (4), vol. 2 (1876), p. 430.

4. T. Levi-Civita, Scie e leggi di resistenza, Rendiconti del Circolo Matematico di Palermo, 1907, p. 1. 
5. Lord Kelvin, "On the Doctrine of Discontinuity of Fluid Motion in Connection with the Resistance against a Solid Moving through a Fluid," Mathematical and Physical Papers, vol. 4 (1910), p. 215. Also in Nature, vol. 50 (1894), p. 524.

6. G. Greenhill, Report on the Theory of a Streamline Past a Plane Barrier, . . , British Aeronautical Research Committee, Reports and Memoranda, no. 19, 1910.

7. —- The Dynamics of Mechanical Flight, London, 1912.

8. H. Villat, Sur la résistance des fluides, Annales Scientifique de l'École Normale Supérieure, (3), vol. 28 (1911), p. 203, and ibid. vol. 31 (1914), p. 455.

9. V. Valcovici, Über diskontinuierliche Fluissigkeitsbewegungen mit zwei freien Strahlen, Dissertation, Göttingen, 1913.

10. D. Riabouchinsky, Sur les surfaces de glissement dans les fuides, Bulletin de l'Institute Aérodynamique de Koutchino, vol. 4 (1920), p. 9.

11. - Mouvement initial d'un liquide en contact avec un obstacle d arêtes vives, Comptes Rendus de l'Académie des Sciences, Paris, vol. 172 (1921), p. 521.

12. U. Cisotti, Idrodinamica piana, Milano, 1921.

13. R. Thirry, Sur les solutions multiples des problèmes d'hydrodynamique relatives aux mouvements glissants, Annales Scientifique de l'École Normale Supérieure, (3), vol. 38 (1921), p. 229.

14. D. Riabouchinsky, Recherches d'Hydrodynamique, Thìses, Paris, 1922. See also, On some cases of two-dimensional fuid motions, Proceedings of the London Mathematical Society, (2), vol. 25 (1926), p. 185.

15. A. Weinstein, Sur l'unicité des mouvements glissants, Comptes Rendus de l'Académie des Sciences, Paris, vol. 176 (1923), p. 493. Ein hydrodynamischer Unitätssatz, Mathematische Zeitschrift, vol. 19 (1924), p. 265.

16. G. Hamel, Ein hydrodynamischer Unitätssatz, Proceedings of the Second International Congress of Applied Mechanics (Zürich, 1926), pp. 489-494.

17. D. Riabouchinsky, Sur les singularites des mouvements fluides, ibid., p. 527.

18. S. Brodetzky, Discontinuous fluid motion past curved barriers, ibid., p. 527.

19. H. Weyl, Strahlbildung, nach der Kontinuitätsmethode behandelt, Nachrichten der Gesellschaft der Wissenschaften, Göttingen, 1927, p. 227.

20. L. Rosenhead, Resistance of a barrier in the shape of an arc of a circle, Proceedings of the Royal Society, (A), vol. 117 (1928), p. 417.

21. H. Villat, Leçons sur l'Hydrodynamique, Paris, 1929.

22. C. Schmieden, Die unstetige Strömung um einen Kreiszylinder, IngenieurArchiv, vol. 1 (1929), p. 104.

23. A. Weinstein, Zur Theorie der Fluissigkeitsstrahlen, Mathematische Zeitschrift, vol. 31 (1929), p. 424.

24. D. Riabouchinsky, Sur quelques problèmes généraux relatifs au mouvement et d la résistance des fluides, and Théorie cavitationelle de la résistance des fuides, Proceedings of the Third International Congress of Applied Mechanics (Stockholm, 1930), vol. 1, pp. 137, 148.

25. A. Betz and E. Petterson, Anwendung der Theorie der freien Strahlen, Ingenieur-Archiv, vol. 2 (1931), p. 190.

26. A. Quarleri, Sulla teoria della "scia" nei liquidi perfetti; caso del cilindro rotondo, Rendiconti dell'Accademia dei Lincei, vol. 14 (1931), p. 332.

27. S. Bergmann, Mehrdeutige Lösungen bei Potentialströmungen mit freien Grenzen, Zeitschrift für angewandte Mathematik und Mechanik, vol. 12 (1932), p. 95.

28. A. Weinstein, Sur les sillages provoqués par des arcs circulaires, Rendiconti dell'Accademia dei Lincei, vol. 17 (1933), p. 83. 
29. Sur les points de detachement des lignes de glissement, Comptes Rendus de l'Académie des Sciences, Paris, vol. 196 (1933), p. 324.

30. K. Friedrichs, Über ein Minimumproblem für Potentialströmungen mit freiem Rande, Mathematische Annalen, vol. 109 (1933), p. 64.

31. J. Leray and A. Weinstein, Sur un problème de representation conforme posé par la theorie de Helmholtz, Comptes Rendus de l'Académie des Sciences, Paris, vol. 198 (1934), p. 430.

32. B. Demtchenko, Problèmes Mixtes Harmoniques en Hydrodynamique des Fluides Parfaits, Paris, 1933, where the:e are extensive bibliographies of this subject.

33. G. Hamel, Über das d'Alembertsche Paradoxon (Abhandlungen zur Hydrodynamik, III.) Zeitschrift für angewandte Mathematik und Mechanik, vol. 15 (1935), pp. 52-55.

34. A. Vitols, Beitrag zur Frage des vakuumlosen Dammprofils, Wasserkraft und Wasserwirtschaft, vol. 31 (1936), pp. 207-212.

35. A. Weinstein, Les conditions aux limites introduites par l'hydrodynamique, L'Enseignement Mathématique, vol. 35 (1936), pp. 107-125.

36. A. Fischer, Beitrag zur Ermittelung des vakuumlosen Dammprofils nach dem Verfahren von A. Witols, Wasserkraft und Wasserwirtschaft, vol. 32 (1937), pp. 281-284.

\section{WAVES WITH FINITE AMPLITUDES}

1. G. G. Stokes, On the theory of oscillatory waves, Transactions of the Cambridge Philosophical Society, vol. 8 (1847). Also Papers, vol. 1, p. 197.

2. Lord Rayleigh, On waves, Philosophical Magazine, (5), vol. 1 (1876), p. 257. Also Papers, vol. 1, p. 251.

3. J. McCowan, On the solitary wave, ibid., vol. 32 (1891), p. 45.

4. A. G. M. Michell, The highest waves in water, ibid., vol. 36 (1893), p. 430.

5. J. McCowan, On the highest wave of permanent type, ibid., vol. 37 (1894), p. 351.

6. D, J. Korteweg and De Vries, On the change of form of long waves advancing in a rectangular canal and on a new type of long stationary waves, ibid., vol. 39 (1895), p. 422.

7. Lord Rayleigh, Periodic waves in deep water advancing without change of type, ibid., (6), vol. 21 (1911), pp. 183-187.

8. J. R. Wilton, On the highest waves in deep water, ibid., (6), vol. 26 (1913), pp. 1053-1058.

9. W. Burnside, On the periodic irrotational waves at the surface of deep water, Proceedings of the London Mathematical Society, (2), vol. 15 (1916), pp. 26-30.

10. Lord Rayleigh, On the periouic irrotational waves at the surface of deep water, Philosophical Magazine, (6), vol. 33 (1917), pp. 381-389.

11. T. H. Havelock, Periodic irrotational waves of finite height, Proceedings of the Royal Society, vol. 95 (1918), pp. 38-51.

12. T. Levi-Civita, Determination rigoureuse des ondes permanentes d'ampleur finie, Mathematische Annalen, vol. 93 (1925), pp. 264-314.

13. K. J. Struik, Determination rigoureuse des ondes irrotationelles périodiques dans un canal a profondeur finie, ibid., vol. 95 (1926), pp. 595-634.

14. A. Weinstein, Sur la vitesse de propagation de l'onde solitaire, Rendiconti dell'Accademia dei Lincei, (6), vol. 3 (1926), pp. 463-468.

15. M. L. Dubreil-Jacotin, Sur la détermination rigoureuse des ondes périodiques d'ampleur finie, Journal de Mathématique, vol. 13 (1934), pp 217-291. 


\section{VISCOUS FLUIDS}

1. C. L. Navier, Mémoire sur les lois du mouvement des fluides, Mémoire de l'Académie des Sciences, 1822.

2. G. G. Stokes, On the theories of the internal friction of fluids in motion, Transactions of the Cambridge Philosophical Society, vol. 8 (1845), or Papers, vol. 1. On the effect of the internal friction of fluids on the motion of pendulums, ibid., vol. 9 (1851), or Papers, vol. 3, p. 48.

3. O. Reynolds, On the dynamical theory of incompressible viscous fuids and the determination of the criterion, Philosophical Transactions of the Royal Society, London, 1895. Or Collected Papers, vol. 2, p. 535.

4. L. Prandtl, Über Flïssigkeitsbewegung bei sehr kleiner Reibung, Proceedings of the Third International Congress for Mathematics (1904), pp. 484-491.

5. H. Blasius, Grenzschichten in Flüssigkeiten mit kleiner Reibung, Zeitschrift für Mathematik und Physik, vol. 56 (1908), pp. 1-37.

6. K. Hiemenz, Die Grenzschicht an einem in den gleichförmigen Flüssigkeitsstrom eingetauchten geraden Kreiszylinder, Dinglers polytechnisches Journal, vol. 326 (1911), pp. 321-324.

7. C. W. Oseen, Über die Stokessche Formel und über eine verwandte Aufgabe in der Hydrodynamik, Archiv för matematik, astronomi och fysik, vol. 6 (1910), vol. 7 (1911), and vol. 9 (1913).

8. G. Hamel, Spiralförmige Bewegungen zäher Flüssigkeiten, Jahresbericht der Deutschen Mathematiker-Vereinigung, vol. 25 (1916), pp. 34-60.

9. Th. von Kármán, Über laminare und turbulente Reibung, Zeitschrift für angewandte Mathematik und Mechanik, vol. 1 (1921), pp. 233-252.

10. K. Pohlhausen, Zur näherungsweisen Integration der Differentialgleichung der laminaren Grenzschicht, ibid., vol. 1 (1921), pp. 257-261. 431.

11. R. von Mises, Bemerkungen zur Hydrodynamik, ibid., vol. 7 (1927), pp. 425-

12. C. W. Oseen and B. Faxen, "Flüssigkeits bewegung mit Reibung," Chapter XXI of Frank Mises, Die Differential und Integralgleichungen der Mechanik und Physik, vol. 2, 1927.

13. C. W. Oseen, Neuere Methoden und Ergebnisse der Hydrodynamik, Leipzig, 1927.

14. L. Lichtenstein, Grundlagen der Hydromechanik, Springer, Berlin, 1929.

15. V. M. Falkner and Sylvia W. Skan, Some Approximate Solutions of the Boundary Layer Equations, British Aeronautical Research Committee, Reports and Memoranda, no. $1314,1930$.

16. J. J. Green, Viscous Layer Associated with a Circular Cylinder, British Aeronautical Research Committee, Reports and Memoranda, no. 1313, 1930.

17. Th. von Kármán and C. B. Millikan, On the Theory of Laminar Boundary Layer Involving Separation, National Advisory Committee for Aeronautics Technical Report, no. 504, 1934.

18. L. Howarth, On the Calculation of Steady Flow in the Boundary Layer Near the Surface of a Cylinder in a Stream, British Aeronautical Research Committee, Reports and Memoranda, no. 1632, 1935.

19. D. R. Hartree, On an equation occurring in Falkner and Skan's approximate treatment of the equations of boundary layer, Proceedings of the Cambridge Philosophical Society, vol. 33 (1937), pp. 223-239.

20. D. R. Hartree and J. R. Womersley, A method for the numerical or mechanical 
solutions of certain types of partial differential equations, Proceedings of the Royal Society, (A), vol. 161 (1937), pp. 353-366.

21. S. Goldstein and others, Modern Developments in Fluid Dynamics, vols. 1 and 2, Oxford, 1938.

\section{COMPRESSIBLE FLUIDS}

1. B. Riemann, Über die Fortpflanzung ebener Luftwellen von endlicher Schwingungsweite, Abhandlungen der Gesellschaft der Wissenschaften zu Göttingen, mathematisch-physikalischer Klasse, vol. 8 (1860), p. 43. Or Gesammelte Werke, 1876, p. 144.

2. H. Hugoniot, Sur la propagation du mouvement dans les corps et spécialement dans les gaz parfaits, Journal de l'École Polytechnique, vol. 58 (1889), p. 80.

3. A. Chaplygin, Gas Jets, Scientific Memoirs of the University of Moscow, 1902.

4. Th. Meyer, Über zweidimensionale Bewegungsvorgänge in einem Gas, das mit Überschallgeschwindigkeit strömt, Verein der Deutschen Ingenieure, Forschungsheft, no. 62, 1908.

5. O. Janzen, Beitrag zu einer Theorie der stationären Strömung kompressibler Flïssigkeiten, Physikalische Zeitschrift, vol. 14 (1913), p. 639.

6. Lord Rayleigh, On the flow of compressible fluid past an obstacle, Philosophical Magazine, (6), vol. 32 (1916), p. 1. Or Papers, vol. 6, p. 402.

7. H. Glauert, The effect of compressibility on the lift of aerofoils, Proceedings of the Royal Society, (A), vol. 118 (1927), p. 113.

8. G. I. Taylor and C. F. Sharman, A mechanical method for solving problems of flow in compressible fluids, ibid., vol. 121 (1928), p. 194.

9. H. Bateman, Notes on a differential equation which occurs in the two-dimensional motion of a compressible fluid and the associated variational problem, ibid., vol. 125 (1929), p. 598.

10. A. Busemann, Drücke auf kegelförmige Spitzen bei Bewegung mit Überschallgeschwindigkeit, Zeitschrift für angewandte Mathematik und Mechanik, vol. 9 (1929), p. 496.

11. L. Prandtl and A. Busemann, Nährungsverfahren zur zeichnerischen Ermittelung von ebenen Strömungen mit Überschallgeschwindigkeit, Stodola Festschrift, Zürich, 1929, p. 499.

12. A. Busemann, Verdichtungstösse in ebenen Gasströmungen, "Vorträge aus dem Gebiete der Aerodynamik," Aachen, 1929, published by Gilles, Hopf and von Kármán, Berlin, 1930, p. 162.

13. L. Prandtl, Über Strömungen, deren Geschwindigkeiten mit der Schallgeschwindigkeit vergleichbar sind, Journal of the Aeronautical Research Institute of the University of Tokyo, no. 6 (1930), p. 14.

14. G. I. Taylor, The Flow of Air at High Speeds Past Curved Surfaces, British Aeronautical Research Committee, Reports and Memoranda, no. 1381, 1930.

15. L. Crocco, Su di un valore massimo del coefficiente di transmissione del calore da una lamina piana a un fluido scorrente, Rendiconti dell'Accademia dei Lincei, vol. 14 (1931), pp. 490-496.

16. S. G. Hooker, The Flow of a Compressible Fluid in the Neighbourhood of the Throat of a Constriction in a Circular Wind Channel, British Aeronautical Research Committee, Reports and Memoranda, no. 1429, 1931.

17. G. Braun, Die ebene kompressible Potentialströmung als Variations- und Eigenwertproblem, Annalen der Physik, (5), vol. 15 (1932), p. 645.

18. B. Demtchenko, Sur les mouvements lents des fuids compressibles, Comptes Rendus de l'Académie des Sciences, Paris, vol. 194 (1932), p. 1218. 
19. - Wariation de la résistance aux faibles vitesses sous l'influence de la compressibilitê, ibid., vol. 194 (1932), p. 1720.

20. Th. von Kármán and N. B. Moore, Resistance of slender bodies moving with supersonic velocities with special reference to projectiles, Transactions of the American Society of Mechanical Engineers, vol. 54 (1932), p. 303.

21. L. Poggi, Campo di velocitd in una corrente piana di fluido compressibile, L'Aerotecnica, vol. 12 (1932), p. 1579.

22. D. Riabouchinsky, Mouvement d'un fluide compressible autour d'un obstacle, Comptes Rendus de l'Académie des Sciences, Paris, vol. 194 (1932), p. 1215.

23. G. I. Taylor and J. W. Maccoll, The air pressure on a cone moving at high speeds, Proceedings of the Royal Society, (A), vol. 139 (1933), pp. 278 and 298.

24. L. Poggi, Campo di velocita in una corrente piana di fluido compressibile. Parte II: Caso di profili ottenuti con representazione conforme dal cerchio ed in particolare dei profili Joukowski, L'Aerotecnica, vol. 14 (1934), p. 532.

25. A. Busemann, Gasströmung mit laminarer Grenzschicht entlang einer Platte, Zeitschrift für angewandte Mathematik und Mechanik, vol. 15 (1935), p. 23.

26. —- Aerodynamischer Auftrieb bei Überschallgeschwindigkeit, Luftfahrtforschung, vol. 12 (1935), pp. 210-220.

27. C. Ferrari, Determinazione della pressione sopra solidi di rivoluzione a prora acuminata disposti in deriva in corrente di fluido compressibile a velocitd ipersonora, Atti della Accademia delle Scienze di Torino, vol. 72 (1936).

28. L. Prandtl, Theorie des Flugzeugtragflïgels im zusammendruckbaren Medium, Luftfahrtforschung, vol. 13 (1936), pp. 313-319.

29. H. Schlichting, Tragflügeltheorie bei Überschallgeschwindigkeit, Luftfahrtforschung, vol. 13 (1936), pp. 320-335.

30. A. Busemann, Hodographenmethode der Gasdynamik, Zeitschrift für angewandte Mathematik und Mechanik, vol. 17 (1937), pp. 73-79.

31. L. Crocco, Eine neue Stromfunktion für die Erforschung der Bewegung der Gase mit Rotation, ibid., vol. 17 (1937), pp. 1-7.

32. G. Hamel, Eine besondere Art von Luftbewegung, Forschungsarbeiten auf dem Gebiet des Ingenieurwesens, vol. 8 (1937), pp. 13-14.

33. I. Imai, On the flow of a compressible fluid past a circular cylinder, Proceedings of the Physico-Mathematical Society of Japan, (3), vol. 20 (1938), pp. 635-645.

34. C. Kaplan, Two-Dimensional Subsonic Compressible Flow Past Elliptic Cylinders, National Advisory Committee for Aeronautics Technical Report, no. 624, 1938.

35. Th. von Kármán and H. S. Tsien, Boundary layer in compressible fluids, Journal of the Aeronautical Sciences, vol. 5 (1938), pp. 227-232.

36. H. S. Tsien, Supersonic flow over an inclined body of revolution, ibid., vol. 5 (1938), pp. $480-483$.

37. — , Two-dimensional subsonic flow of compressible fuids, ibid., vol. 6 (1939), pp. 399-407.

38. L. Crocco, Una caratteristica trasformazione delle equazioni dello strato limite nei gas, Atti di Guidonia, no. 7 (1939).

39. H. Görtler, Zum Übergang von Unterschall- zu Überschallgeschwindigkeiten in Dïsen, Zeitschrift für angewandte Mathematik und Mechanik, vol. 19 (1939), pp. 325-337.

California Institute of Technology 